\title{
Local supercluster dynamics: external tidal impact of the PSCz sample traced by optimized numerical least action method ${ }^{\star}$
}

\author{
E. Romano-Díaz ${ }^{1,2}$, E. Branchini ${ }^{3}$, and R. van de Weygaert ${ }^{1}$ \\ 1 Kapteyn Astronomical Institute, University of Groningen, PO Box 800, 9700 AV Groningen, The Netherlands \\ 2 Racah Institute of Physics, Hebrew University, 91904 Jerusalem, Israel \\ e-mail: emilio@phys.huji.ac.il \\ ${ }^{3}$ Dipartimento di Fisica, Universita' Degli Studi di Roma Tre, via della Vasca Navale 84, 00146 Roma, Italy
}

\section{Received 14 September 2004 / Accepted 20 April 2005}

\begin{abstract}
We assess the extent to which the flux-limited IRAS PSC $z$ redshift survey encapsulates the complete or major share of matter inhomogeneities responsible for the external tidal forces affecting the peculiar velocity flow within the Local Supercluster and its immediate surroundings. We here investigate this issue on the basis of artificially constructed galaxy catalogs. Two large unconstrained $N$-body simulations of cosmic structure formation in two different cosmological scenarios form the basis of this study. From these $N$-body simulations a set of galaxy mock catalogs is selected. From these a variety of datasets is selected imitating the observational conditions of either the local volume-limited Local Supercluster mimicking NBG catalog or the deeper magnitude-limited PSC $z$ catalog. The mildly nonlinear dynamics in the "mock" Local Supercluster and PSC $z$ velocities are analyzed by means of the Least Action Principle technique in its highly optimized implementation of the Fast Action Method. By comparing the velocities in these reconstructions with the "true" velocities of the corresponding galaxy mock catalogs we assess the extent and nature of the external tidal influence on the Local Supercluster volume. We find that the dynamics in the inner $30 \mathrm{~h}^{-1} \mathrm{Mpc}$ volume is strongly affected by the external forces. Most of the external forces can be traced back to a depth of no more than $100 \mathrm{~h}^{-1} \mathrm{Mpc}$. This is concluded from the fact that the FAM reconstructions of the $100 h^{-1} \mathrm{Mpc}$ PSC $z$ volume appear to have included most gravitational influences. In addition, we demonstrate that for all considered cosmological models the bulk flow and shear components of the tidal velocity field generated by the external distribution of PSC $z$ galaxies provides sufficient information for representing the full external tidal force field.
\end{abstract}

Key words. cosmology: theory - cosmology: large-scale structure of Universe - methods: numerical - surveys

\section{Introduction}

Migration flows of cosmic matter are one of the major physical manifestations accompanying the emergence and growth of structure out of the virtually homogeneous primordial Universe. The cosmic flows displace matter towards the regions where ever more matter accumulates, ultimately condensing into the objects and structures we observe in the Universe.

Within the gravitational instability scenario of structure formation, the displacements are the result of the cumulative gravitational force exerted by the inhomogeneous spatial matter distribution of continuously growing density surpluses and deficits throughout the Universe. This establishes a direct causal link between gravitational force and the corresponding peculiar velocities. Given a suitably accurate measurement of peculiar velocities within a well-defined "internal" region of space, $V_{\text {int }}$, we may invert these velocities and relate them to the inducing gravitational force. Hence, the source of the measured motions

* Sections 5-7, associated Figs. 3-11 and Tables 2 and 3 are only available in electronic form at http: //www . edpsciences.org/. may be traced and possibly even reconstructed. In principle, it may even allow us to infer the total amount of mass involved and thus estimate the value of the cosmological density parameter $\Omega_{\mathrm{m}}$ and other fundamental cosmological parameters.

The practical execution of such studies of cosmic velocity flows is ridden by various complicating factors. One major complication is that the cosmic regions in which peculiar velocities have been determined to sufficient accuracy may have a substantially smaller size than what may be deemed appropriate for a dynamically representative volume. Ideally, in order to account for almost the complete flow in our local cosmic neighbourhood we should have probed the density field in a sufficiently large cosmic volume. This should involve a region of space substantially superseding that of the characteristic scale of the largest coherent structures in the Universe. Only then the magnitude of the gravitational influence of inhomogeneities at larger distances will represent a negligible contribution and, as well, start to even out against each other.

The size of this dynamically effective volume depends sensitively on the structure formation scenario which is prevailing in our Universe. Hence, it will be closely affiliated to the 
spatial distribution, characteristic size and coherence scale of cosmic structures, and its size will therefore be in the order of the scale of the largest pronounced structures in the Universe. Within the conventional structure formation models, based on Gaussian initial density and velocity fields, this is fully specified through the scenario's fluctuations power spectrum $P(k)$. When the power spectrum involves a substantial large-scale component and the survey volume is rather limited we have to be aware of significant external influences. Although not yet exactly determined, observational evidence suggests its size to be in the range of $\approx 100-200 h^{-1} \mathrm{Mpc}$ (where $h$ denotes the Hubble constant in units of $100 \mathrm{~km} \mathrm{~s}^{-1} \mathrm{Mpc}^{-1}$ ).

An equally important consideration concerns the spatial resolution at which the velocity field is studied. Available samples of galaxy peculiar velocities extend out to reasonable depth of $\approx 60 h^{-1} \mathrm{Mpc}$. Yet, they involve a rather coarsely and inaccurately sampled cosmic velocity field. By absence of precise distance estimators, more accurately and densely sampled velocity information is therefore mostly confined to a rather limited region in and around the Local Supercluster [LS]. As a consequence, most analyses of large-scale cosmic flows are necessarily confined to spatial scales at which the evolving cosmic structures are still residing in a linear phase of development. The dynamics in more advanced stages of cosmic structure formation are as yet poorly constrained by measurements.

\subsection{Cosmic force fields and supercluster dynamics}

In this work we wish to extend the analysis of cosmic flows to the more advanced evolutionary stages pertaining within supercluster regions. Only within the local cosmic neighbourhood of our Local Supercluster, the quality, quantity and spatial coverage of the peculiar velocity data are sufficiently good to warrant an assessment of the cosmic velocity field and the corresponding dynamics at a sufficiently high spatial resolution. On these quasi-linear or mildly nonlinear scales we hope to find traces of the onset towards the more advanced stages of cosmic structure formation. In order for this to yield a meaningful and successful analysis, two major questions have to be addressed. Both form the main focus of this contribution.

The first issue, that of the rather restricted sample volume, constitutes the major incentive behind this work. The volume of the galaxy catalog that best samples our local cosmic neighbourhood, the Nearby Galaxy Catalog (Tully 1988, hereafter (NBG)), is certainly substantially smaller than what may be considered dynamically representative. Any analysis of the (internal) velocity field in our Local cosmic neighbourhood should therefore take into account the impact of external gravitational influences.

We focus on two related problems. In the first place, there is the need to quantify the effect of neglecting the external gravitational influence $\boldsymbol{g}_{\text {ext }}$ when modeling the cosmic velocity field on scales comparable to that of the Local Supercluster. Various studies have attempted to determine cosmological parameters on the basis of a comparison between modelled versus measured velocity field in the Local Universe (Tonry \& Davis 1981; Tully \& Shaya 1984; Shaya et al. 1995;
Tonry et al. 2000). For this it is crucial to understand in how far local density perturbations may account for the local peculiar gravity field within the Local Supercluster. Directly related to this is the need to have a sufficiently accurate description of the external force field $\boldsymbol{g}_{\text {ext }}$, in terms of its nature and spatial extent, in order to properly model the total measured gravity field $\boldsymbol{g}_{\text {tot }}$. For studies intent on a comparison of modelled versus observed peculiar velocities on scales larger than the Local Supercluster this is an essential requirement (Faber \& Burstein 1988; Han \& Mould 1990; Yahil et al. 1991; Webster et al. 1997; Branchini et al. 1999). Similar considerations are equally relevant for the inverse problem, in which one attempts to infer the external gravitational influence $\boldsymbol{g}_{\text {ext }}$ from peculiar velocities measured within the Local Supercluster (e.g. Lilje et al. 1986; Lynden-Bell \& Lahav 1988; Kaiser 1991; Hoffman et al. 2001). Indeed, both problems concerning the restricted galaxy sample volume have figured prominently in previous cosmic velocity field studies and were addressed in a variety of publications. However, usually these tend to discard the fact that the local cosmic region in which we have access to high quality velocity data has already reached an advanced quasi-linear dynamical state.

Referring to the latter, the second major issue concerns the innovative way in which we evaluate the dynamical state of superclusters. These structures reside in a mildly nonlinear evolutionary stadium, having evolved significantly beyond their initial linear phase. Unlike the vast majority of previous studies, we seek to probe into the more detailed and informative kinematic aspects of these structures. A conventional linear analysis will not be able to provide an adequate description, and for the most evolved circumstances not even the Zel'dovich approximation (Zel'dovich 1970) may be expected to do so. In order to be able to optimally exploit the available velocity information - without suffering the loss of valuable high-resolution information through a filtering process - we apply the Least Action Principle (LAP) formalism (Peebles 1989) for dealing with the individual galaxy velocities. To that end, an optimal implementation developed by Nusser \& Branchini (2000, hereafter (NB)), the Fast Action Minimization (FAM) proved an essential tool.

Elaborating on the first issue of the external gravitational influence, one of the as yet undecided issues is the extent to which a LAP analysis of a cosmological self-gravitating system is dependent on a proper representation of the external gravitational influence. Various strategies have been followed, ranging from a complete neglect of external forces (Peebles 1989), or taking account of the influence of merely a few nearby objects (Peebles 1989, 1990; Dunn \& Laflamme 1993, 2001), towards methods involving approximate descriptions of external influences. The latter mostly incorporated the wider external influence through a frozen, linearly evolving, external tidal field estimated on the basis of the present-day locations of an extended sample of objects deemed representative for the external matter distribution (e.g. Shaya et al. 1995; Schmoldt \& Saha 1998; Sharpe et al. 2001). This study does include the influence of force fields, but does so in a fully systematic and self-consistent fashion, enabled by the FAM method to take into account the evolution of the full sample of external matter concentrations. 
The principal conclusion of our study is that the gravitational forces exacted by the matter inhomogeneities encapsulated by the IRAS-PSC $z$ redshift survey sample (Saunders et al. 2000) are indeed able to account for all motions within our local Universe. In addition, we demonstrate that its external influence may almost exclusively be ascribed to the bulk and shear flow components.

\subsection{Strategy}

This study is based on a number of artificial galaxy samples mimicking the properties of genuine catalogs. They consist of several well-defined and well-selected model catalogs of galaxies and galaxy peculiar velocities. These mock samples are extracted from a set of extensive $N$-body simulations: for the nearby Universe models they adhere to the characteristics of the Nearby Galaxy Catalog, for the deep galaxy redshift samples they are modelled after the IRAS PSC $z$ catalog. These model samples allow us to thoroughly investigate the various strategies forwarded for a successful and conclusive analysis.

Two sets of realistic mock catalogs of galaxies are extracted. The first set, the "local" one, is meant to mimic the mass distribution within the LS as traced by galaxies in the Nearby Galaxy Catalog of Tully (1988). It consists of a volume-limited galaxy sample within a (spherical) interior region with radius $30 h^{-1} \mathrm{Mpc}$. Each of these interior samples is embedded within a larger mock sample, the "extended" sample which covers a larger cosmic region. In addition to the interior volume-limited sample in the inner $30 h^{-1} \mathrm{Mpc}$ they contain an enclosing outer flux-limited sample covering the surrounding spherical region located between $30 h^{-1} \mathrm{Mpc}<$ $x<100 h^{-1} \mathrm{Mpc}$. This exterior sample mimics a flux-limited galaxy catalog whose characteristics are modelled after the IRAS PSC $z$ sample.

For both the "local" and "extended" mock samples we model the peculiar velocity field at the positions of the particles in the "local" cosmic region, i.e. for the objects out to a radius $x<30 h^{-1} \mathrm{Mpc}$. These model predictions result from the application of Fast Action Minimization method (Nusser \& Branchini 2000). As our FAM reconstruction procedure only takes into account the gravitational forces between the particles in the mock samples - i.e. does not include contributions from outside objects - the differences in predicted velocities between the "local" and "extended" samples will reflect the influence of the mass concentrations in the surrounding region $30 h^{-1} \mathrm{Mpc}<r<100 h^{-1} \mathrm{Mpc}$. The comparison with the corresponding $N$-body velocities, representing the "real" velocities, will inform us in how far "sky-covering" samples of galaxies with a depth of $\approx 100 \mathrm{~h}^{-1} \mathrm{Mpc}$ may be expected to represent a proper cosmic region as far as its dynamics are concerned. The strategy of analyzing and comparing the velocity models obtained from the small "local" mock catalogs, the large "extended" mock catalogs, and the "real" velocities in the original $\mathrm{N}$-body simulations, will yield a solid understanding of the effect of neglecting the externally induced peculiar gravitational acceleration $\boldsymbol{g}_{\text {ext }}$ (see Eq. (1)).
When analyzing a dataset of galaxy peculiar velocities in the local Universe. The analysis of the larger PSC $z$ mimicking catalogs should elucidate if and to what extent such samples will be able to account for $\boldsymbol{g}_{\text {ext }}$. If the galaxies in these samples indeed appear to be responsible for the major share of the external forces, we may feel reassured to use the PSC $z$ sample of galaxies for a proper representation of $\boldsymbol{g}_{\text {ext }}$.

One aspect of this question concerns the investigation of the question whether the external tidal influence may be explicitly framed in an analytical approximation consisting of a dipolar and quadrupolar term. Our fully self-consistent FAM reconstructions, in which the "extended" mock catalogs are processed with the inclusion of all external matter concentrations, enable us to estimate the bulk and shear components in the induced "local" galaxy motions. By comparing the resulting velocity fields in the "local" and "extended" samples we will be able to judge the quality of the approximate methods, and quantify and investigate the possible presence of systematic trends throughout the "local" cosmos.

To account for possible systematic effects due to global cosmology, the mock galaxy catalogs are extracted from $N$-body simulations in two different cosmic structure formation scenarios. One involves a $\Lambda C D M$ Universe with a characteristic largescale dominated power spectrum, while the other concerns a $\tau C D M$ cosmology. The more small-scale dominated character of the latter leads to a different character of its gravitational field fluctuations, the smaller coherence scale of the density field fluctuations yielding a comparatively smaller influence of the external (quadrupolar) tidal field (the induced bulk flows are similar, as the smaller $\tau C D M$ fluctuations are exactly compensated by the larger mass involved). The resulting comparisons of FAM velocity field reconstructions are expected to reflect these velocity field differences.

In the end, this study of artificial galaxy samples should allow us to appreciate the manifestations of the real physical effects we wish to grasp. In this, we also should learn how to deal with the complications due to the host of measurement uncertainties which beset the observational data. The scope is to quantify the systematic errors which might have affected similar, local, comparisons based on real data and to judge whether the information on the external mass distribution available to these analyses is indeed sufficient to account for $\boldsymbol{g}_{\text {ext }}$.

\subsection{Outline}

In the next section, we will elaborate on the astrophysical background of this study, the study of velocity flows on cosmological scales, and in particular the issue of internal and external gravitational influences. Ensuingly, we address the specific problem of treating the dynamics and related cosmic motions within mildly nonlinear structures such as the Local Supercluster. This brings us to a brief exposition on the LAP analysis for dealing with the complications of mildly nonlinear orbits and the technical issue of the FAM technique which allows us to apply this to a system composed of many objects. Special emphasis is put on the inclusion of external gravitational influences within the LAP/FAM formalism. In Sect. 4 we 
describe the cosmological setting of the simulations on which this study has been based. As a guidance towards interpreting our calculations, we address a variety of theoretical aspects and predictions concerning cosmic velocity fields in these cosmological scenarios. The basis of this work is the set of two "parent" $N$-body simulations and the mock catalogs extracted from these simulations, forty in total. They are presented in Sect. 5. In the subsequent sections we present the results obtained from the various FAM computations. In Sect. 6 we analyze the velocity vector maps for the FAM reconstructions. These maps allow a direct and visually illuminating appreciation of the effects we wish to address. This is followed by a first quantitative assessment in Sect. 7. This consists of a comparison between the FAM velocity field reconstructions of the Local Supercluster volume $\left(r<30 h^{-1} \mathrm{Mpc}\right)$, the FAM reconstructions for the corresponding PSC $z$ sample and the complete "real world" $N$-body velocity field. The comparison is mainly based on a point-by-point evaluation through scatter diagrams of velocity-related quantities. To encapsulate these results into a spatially coherent description of the large scale external velocity and gravity field, in Sect. 8 we turn to a decomposition of the peculiar velocity field into multipolar components. In particular, we demonstrate that a restriction to its dipolar and quadrupolar components, i.e. the bulk flow and velocity shear, does represent a good description. Thus having looked at the issue of cosmic velocity fields from different angles, the summary of Sect. 9 will focus on the repercussions of our analysis and its relation to the study of the (relatively nearby) surrounding matter distribution. On the basis of these conclusions we provide a description of the various projects which follow up on this work, together with some suggestions for additional future work.

\section{Cosmic flows: probes of cosmic matter distribution}

\subsection{The large-scale Universe: linear flows}

Over the past two decades a major effort has been directed towards compiling large samples of galaxy peculiar velocities. These samples made it possible to obtain a rather impressive view of cosmic dynamics on scales $\gtrsim 10 h^{-1} \mathrm{Mpc}$. In particular the Mark III catalog, with an effective depth $\approx 60 h^{-1} \mathrm{Mpc}$, stands as a landmark achievement (Willick et al. 1997a; also see Dekel 1994; and Strauss \& Willick 1995). Further progress has been enabled by the assembly of additional and partially complementary samples of galaxy peculiar velocities, of which the SFI late-type galaxy and ENEAR early-type galaxy samples are noteworthy examples. The SFI Catalog of Peculiar Velocities of Galaxies (Giovanelli et al. 1997a,b; Haynes et al. 1999a,b) consists of around 1300 spiral galaxies with $I$-band Tully-Fisher (TF) distances, out to $c z<7500 \mathrm{~km} \mathrm{~s}^{-1}$. The ENEAR sample (da Costa et al. 2000) is an equivalent sample of around 1600 early-type galaxies, out to a distance $c z<$ $7000 \mathrm{~km} \mathrm{~s}^{-1}$, with $D_{n}-\sigma$ distance estimates available for nearly all of them. Tracing cosmic motions over larger volumes of space is a rather more cumbersome affair and attempts to do so are mainly based upon the peculiar motions of galaxy clusters. The claim of a puzzlingly large flow over scales of $150 h^{-1} \mathrm{Mpc}$ by Lauer \& Postman (1994) could not be corroborated. Nonetheless, flows on such large scales may indeed be a reality, as has been inferred from the far better defined "Streaming Motions of Abell Clusters" (SMAC) sample of Hudson et al. (2001). They did recover a bulk flow in the order of $687 \pm 203 \mathrm{~km} \mathrm{~s}^{-1}$, of which $225 \mathrm{~km} \mathrm{~s}^{-1}$ may arise from sources at a distance larger than $100 \mathrm{~h}^{-1} \mathrm{Mpc}$ (Hudson et al. 2004). One prime objective of most analyses of these large samples of peculiar velocities has been the determination of the cosmological mass density parameter $\Omega_{\mathrm{m}}$ (Davis et al. 1996; Willick et al. 1997b; Willick \& Strauss 1998; Nusser et al. 2000; Branchini et al. 2001). Such assessments are based on a comparison of observed velocities to a model velocity field. A basic requirement for obtaining self-consistent estimates of $\Omega_{\mathrm{m}}$ is that the velocity samples concern a "representative" volume of space.

However, even while such studies appear to succeed in attuning the large-scale matter distributions and velocity fields in a reasonably self-consistent fashion, doubts remain with respect to a variety of practical and systematic problems. Firstly, in these comparisons the random errors on the observed velocities are substantial, much larger than those in the structure formation models. Considerable effort has been directed towards quantifying and minimizing errors on the observed peculiar velocities (e.g. Dekel 1994; Strauss \& Willick 1995, and references therein). These involve random measurement errors as well as more subtle systematic, yet reasonably well understood, errors. Secondly, there remain various systematic effects which have not been addressed and corrected for in an equally convincing fashion. Even though they also tend to play a role with respect to the model predictions they are often overlooked.

A major systematic factor concerns the incomplete information on the spatial mass distribution within the region of the sample itself. This prevents an adequate treatment of artifacts due to the incomplete sky coverage and limited depth of the available samples, and effects systematic errors stemming from luminosity and density effects. These systematic errors are usually accounted for by using large, all-sky redshift surveys, such as the Optical Redshift Survey of Santiago et al. (1995) or the 1.2 Jy and PSC $z$ surveys of IRAS galaxies (Fisher et al. 1995; Saunders et al. 2000). In particular when using IRAS based surveys the effects of incomplete sky coverage are greatly reduced.

Even more problematic for a successful handling of luminosity and density related effects is our incomplete knowledge with respect to the relationship between the observable galaxy distribution and the underlying mass distribution. By absence of a compelling theory of galaxy formation this "galaxy bias" is usually encapsulated in heuristic formulations. The rather ad-hoc and possibly unrealistic or inadequate nature of the latter may seriously affect the significance of the inferred conclusions. Most studies make the simplifying assumption of a galaxy population fairly tracing the underlying density field. This is usually embodied in a global and linear "galaxy bias" factor. A large variety of results suggest that this may be a reasonable approximation on scales in excess of a few Megaparsec. Moreover, while this bias may be 
problematic in the case of early-type galaxies, it has proved to be quite successful with respect to the later type galaxies which figure prominently in IRAS based samples (Verde et al. 2002).

\subsection{Internal and external influences}

Unlike most studies of cosmic flows which seek to assess and analyze the nature and source of dynamical influences within a confined region of space, we try to get an impression of the cosmic dynamics on mildly nonlinear scales of only a few Megaparsec. We focus on the Local Supercluster region and its immediate neighbourhood. The galaxy sample of the NBG catalog is taken to be representative for this region. Because the catalog entails a volume which is substantially smaller than what may be considered dynamically representative, the peculiar velocities of the galaxies are partially due to the gravitational action by outside matter concentrations. That is, the peculiar velocities are not only due to the gravitational force induced by the matter concentrations within the "internal" survey volume $V_{\text {int }}$, but also reflect the gravitational influence by the "external" matter density distribution, $\boldsymbol{g}_{\text {ext }}$. Because it does not constitute a truly representative volume of the Universe, a meaningful dynamic analysis of the Local Universe on the basis of the NBG sample is substantially complicated by its limited depth, which is one of the major systematic problems besetting the analysis of virtually all available surveys of galaxy peculiar velocities. Theoretical models of peculiar velocities nearly always involve the implicit assumption of the mass being homogeneously distributed outside $V_{\text {int }}$, so that its gravitational effect may be neglected. Even in the case of having a sufficiently large volume at one's disposal, this approximation is only valid in the central part of $V_{\text {int }}$, certainly not near its edges.

The distinction between external versus internal gravitational force may be best appreciated by noting that the total (peculiar) gravity field $\boldsymbol{g}_{\text {tot }}(\boldsymbol{x})$ is the netto sum of the individual contributions by all patches of matter throughout the visible Universe. At any position within the internal volume $V_{\text {int }}$, we may then decompose the full gravitational field into an "internally" induced component $\boldsymbol{g}_{\text {int }}$ and an "externally" generated contribution $\boldsymbol{g}_{\mathrm{ext}}$,

$\boldsymbol{g}_{\mathrm{tot}}(x)=g_{\mathrm{int}}(x)+g_{\mathrm{ext}}(x)$.

In this way we have defined the internal gravitational force $\boldsymbol{g}_{\text {int }}$ as the integrated contribution from the density fluctuations $\delta(\boldsymbol{x})$ within the volume $V_{\text {int }}$, while the external gravitational force $\boldsymbol{g}_{\mathrm{ext}}$ concerns the combined gravitational force generated by the density fluctuations outside the realm of $V_{\text {int }}$, so that

$$
\begin{aligned}
\boldsymbol{g}_{\mathrm{tot}}(\boldsymbol{x}, t)= & \frac{3 \Omega H^{2}}{8 \pi} \int_{V_{\text {int }}} \mathrm{d} \boldsymbol{x}^{\prime} \delta\left(\boldsymbol{x}^{\prime}, t\right) \frac{\left(\boldsymbol{x}^{\prime}-\boldsymbol{x}\right)}{\left|\boldsymbol{x}^{\prime}-\boldsymbol{x}\right|^{3}} \\
& +\frac{3 \Omega H^{2}}{8 \pi} \int_{V_{\text {ext }}} \mathrm{d} \boldsymbol{x}^{\prime} \delta\left(\boldsymbol{x}^{\prime}, t\right) \frac{\left(\boldsymbol{x}^{\prime}-\boldsymbol{x}\right)}{\left|\boldsymbol{x}^{\prime}-\boldsymbol{x}\right|^{3}} .
\end{aligned}
$$

The peculiar velocities of galaxies within $V_{\text {int }}$ bear the mark of both the acceleration due to the matter concentrations within the volume itself, $\boldsymbol{g}_{\text {int }}$, as well as that of the combined gravitational influence of the external mass distribution, $\boldsymbol{g}_{\text {ext }}$.
A comparison of predicted internally induced velocities with the observed local velocity field should therefore enable us to infer the magnitude and nature of the external field $\boldsymbol{g}_{\text {ext }}$. This analysis is usually facilitated by the fact that the fine details of the external force contribution are largely negligible. The contributions by the various external matter concentrations to the combined gravitational force mostly average out such that what remains noticeable is mainly confined to the low order components of the multipole decomposition of $\boldsymbol{g}_{\text {ext }}$. This can be most readily appreciated from a description of the external gravitational force field in terms of its successive multipole components. When we expand $\boldsymbol{g}_{\text {ext }}$ around some central location in $V_{\text {int }}$ - here defined to be the origin of the coordinates $\boldsymbol{x}$ - we find that to second order

$g_{\mathrm{ext}, i}(\boldsymbol{x})=g_{\mathrm{bulk}, i}-\sum_{j=1}^{3} \mathcal{T}_{i j} x_{j}$.

In this, we assume that the additional divergence term $\frac{1}{3}(\nabla$. $\left.\boldsymbol{g}_{\text {ext }}\right) x_{i}$ has been embedded into the (zeroth) order monopole term. In essence, it corresponds to a "breathing mode" affecting the "local" Hubble expansion within the volume, and therefore can not possibly be inferred from the local measurement of the internal gravity field $\boldsymbol{g}_{\text {int }}$.

The leading term in the overall external gravitational acceleration is the bulk gravity term $g_{\text {bulk }, i}$. This dipole term constitutes the uniform acceleration of the matter within $V_{\mathrm{int}}$,

$\boldsymbol{g}_{\text {bulk }}=\frac{3 \Omega H^{2}}{8 \pi} \int_{V_{\text {ext }}} \mathrm{d} \boldsymbol{x}^{\prime} \delta\left(\boldsymbol{x}^{\prime}, t\right) \frac{\boldsymbol{x}^{\prime}}{\left|\boldsymbol{x}^{\prime}\right|^{3}}$

Evidently, when considering peculiar velocities relative to the centre of mass inside the volume $V_{\text {int }}$ instead of absolute velocities this constant vector disappears. The first term whose magnitude and configuration is independent of the reference frame is the quadrupolar term $\mathcal{T}_{i j}$.

If the contribution to the (peculiar) gravitational potential by the external mass inhomogeneities is $\phi_{\mathrm{ext}}$, the quadrupolar tidal tensor $\mathcal{T}_{i j}$ is the trace-free part of $\partial^{2} \phi_{\text {ext }} / \partial x_{i} \partial x_{j}$, evaluated at the centre of $V_{i}$. It is determined by the external matter distribution through

$\mathcal{T}_{i j}(t)=\frac{3 \Omega H^{2}}{8 \pi} \int_{V_{\text {ext }}} \mathrm{d} \boldsymbol{x}^{\prime} \delta\left(\boldsymbol{x}^{\prime}, t\right)\left\{\frac{3 x_{i}^{\prime} x_{j}^{\prime}-\left|\boldsymbol{x}^{\prime}\right|^{2} \delta_{i j}}{\left|\boldsymbol{x}^{\prime}\right|^{5}}\right\}$.

The integral expressions for the dipole and quadrupole components of the external gravity field (Eqs. (4) and (5)), illustrate that it is unfeasible to exploit the observed local cosmic velocity field to recover the detailed and complete spatial distribution of the external matter inhomogeneities. On the other hand, it does indicate how it is that we can infer some overall characteristics of the external matter distribution from an analysis of the local velocity field. From this we may extract interesting and significant information on the nature and even distribution of the large scale cosmic matter distribution and set constraints on the values of some of the fundamental cosmological parameters. The pioneering work by Lilje et al. (1986) in which the velocity field of the Local Supercluster was exploited to infer 
the presence of a major external source of gravitational attraction has shown the potential of this approach. Ultimately, it inspired the analysis of Lynden-Bell et al. (1988) that lead to the discovery of the Great Attractor.

\section{Cosmic flows: the mildly nonlinear dynamics of superclusters}

Even though a structure's evolution may have progressed to a dynamical stage at which a first-order description of cosmic velocity fields will no longer be adequate, it may still be possible to find a direct link to the structure's initial configuration. This is in particularly true for the early and mildly nonlinear phases of evolution. The exemplary archetype of a structure in which such mildly nonlinear circumstances are prevalent is that of superclusters, the filamentary or wall-shaped elements of the cosmic foamlike matter distribution.

Over the past two decades intriguing foamlike patterns have gained prominence as a prime characteristic of the cosmic matter distribution. The first indications for the actual existence of a foamlike galaxy distribution were provided by CfA 2 redshift slices (de Lapparent et al. 1986) and established as a universal cosmic pattern with the Las Campanas redshift survey (Shectman et al. 1996). With the arrival of the large recent and ongoing systematic galaxy redshift surveys, the $2 \mathrm{dF}$ galaxy redshift survey $(\approx 250000$ redshifts, Colless et al. 2003; also see e.g. Colless 2004; and Tegmark et al. 2002, for a discussion on clustering in the 2dFGRS) and the Sloan Digital Sky Survey (SDSS, will determine $\approx 1000000$ redshifts, see e.g. Zehavi et al. 2002; and Tegmark et al. 2004, for an overview of present-day status wrt. galaxy clustering), we may hope to have entered the stage in which we will be enabled to explore the formation and the dynamics of these characteristic spatial structures in the cosmic matter distribution. The typical elements of the cosmic foam - filamentary and wall-shaped superclusters - are precisely at the youthful yet mildly nonlinear phase of development mentioned earlier. They were identified as such within the context of Zel'dovich' "pancake" theory of cosmic structure formation (see e.g. Shandarin \& Zel'dovich 1989). The significance of the cosmic foamlike network for the understanding of the process of cosmic structure formation has since been generally recognized. This may be appreciated from the widespread use of the concept of the "cosmic web", coined by Bond et al. (1996) in their study of the dynamics underlying its formation (see Van de Weygaert 2002, for a recent general review).

Mildly nonlinear cosmic features such as superclusters have recently turned their initial co-expansion into a genuine physical contraction (or are on the brink of doing so), marking the emerging structure as a truely identifiable entity. Once it has surpassed this "turn-around" stadium the complexity of its internal kinematics quickly increases. At first moderately, and ultimately dramatically as the virialization process advances, the matter orbits inside the structure become more and more complex. Even in the more moderate early phases of this process, an appropriately sophisticated treatment of the mildly nonlinear dynamics appears to be a necessary requisite for any study based upon kinematic information. In and around emerging nonlinear structures a simple linear analysis for reconstructing initial conditions will therefore no longer suffice. In other words, a sufficiently detailed and profound understanding of the emergence of these key elements in the cosmic matter distribution cannot be obtained without the development of a more elaborate technique for the analysis of cosmic velocity fields.

\subsection{Structure formation: mildly nonlinear dynamics}

A linear analysis simplifies the dynamical evolution of a system into an initial conditions problem. It implies the reconstruction of the primordial density and velocity field by means of a simple linear inversion of the observed matter distribution and galaxy peculiar velocity field. Such an approach may even be followed towards a slightly more advanced stage. The Zel'dovich formalism, a Lagrangian first-order approximation for gravitationally evolving systems, has been remarkably successful in describing the early nonlinear evolution of a supercluster (for a review, see Shandarin \& Zel'dovich 1989). Substantially surpassing its formal linear limitations, it proved to be a highly versatile medium for analyzing and explaining the overall spatial morphology and characteristics of emerging structures. The Zel'dovich approximation elucidated and explained qualitatively the fundamental tendencies of gravitational contraction in an evolving cosmos. Perhaps most noteworthy this concerned the tendency of gravitational collapse to proceed anisotropically, together with its predictive power with respect to location and timescales of the first phase of collapse into planar mass concentrations, "pancakes". This offered the basic explanation for the foamlike morphology of the cosmic matter distribution, stressing its existence many years in advance of its discovery through observational programs to map the galaxy distribution (for an extensive review of various nonlinear approximation schemes seeking to expand upon the Zel'dovich approximation see Sahni \& Coles 1995).

In line with the above, the Zel'dovich approximation proved a highly versatile tool for the analysis of the cosmic matter flows. It was successfully applied to the nonlinear situation of mixed boundary conditions - tested and calibrated using $N$-body simulations - by Nusser et al. (1991) and Nusser \& Dekel (1992). However, its validity remains restricted to the early stages of nonlinearity at which there is still a linear and direct relation between velocity and gravity field. Once matter inside the emerging structures starts to reach densities so high that local interactions become dominant, the Zel'dovich scheme quickly ceases to lose its applicability. Once matter elements start to cross each each others path the interaction between the nonlinear matter concentrations within the realm of the contracting structure will more and more deflect the orbits away from their initial linear trajectory. The linear kinematics of the Zel'dovich approximation will therefore no longer be able to follow the orbits of the matter elements. Higher order approximations based on perturbation theory have been advocated in order to follow such more advanced nonlinear circumstances. However, the improvement over simple first order Zel'dovich approximation turns out to be quite limited and not warranting the effort invested at each successive perturbation 
step. This is particularly so as with the onset of nonlinearity the rate at which successive perturbative orders terms become significant rapidly accelerates.

\subsection{Least action principle in cosmology}

In more advanced nonlinear circumstances we encounter a more generic dynamical situation than a simple initial value problem. Typically, one seeks to compute the velocity field consistent with an observed density structure at the present epoch or, reversely, one deduces the density from the measured peculiar galaxy velocities. In the case of generic systems, the dynamical evolution represents a mixed boundary condition problem. This implies the system to be sufficiently constrained by complementing the incomplete dynamical information regarding the initial conditions with that pertaining to the dynamical state of the system at a different epoch. While $N$-body codes are particularly concerned with the ideal circumstances usually embodied in terms of initial value problems, a different kind of technique needs to be invoked to exploit the typical mixed boundary information yielded by observations.

A more profound and direct exploitation of the available information to follow the physics of such a cosmological nonlinear system was forwarded by Peebles $(1989,1990)$. He pointed out that finding the orbits that satisfy initial homogeneity - and by implication vanishing initial peculiar velocities - and match the (present-day) observed distribution of mass tracers constitutes a mixed-boundary value problem. Such problems lend themselves naturally to an application of Hamilton's principle. This naturally leads to the formulation of the Least Action Principle (also known as "Numerical Action Method"), based on a variational analysis of the action $S$ of an isolated system of $M$ particles, which at a cosmic expansion factor $a(t)$ is given by

$S=\int_{0}^{t_{0}} L \mathrm{~d} t=\int_{0}^{t_{0}} \mathrm{~d} t \sum_{i}\left[\frac{1}{2} m_{i} a^{2} \dot{\boldsymbol{x}}_{i}^{2}-m_{i} \phi\left(\boldsymbol{r}_{i}\right)\right]$,

in which $L$ is the Lagrangian for the orbits of particles with masses $m_{i}$ and comoving coordinates $x_{i}$ and corresponding peculiar gravitational potential $\phi(x)$. For a system of particles interacting by gravity alone, embedded within a uniform cosmological background of density $\rho_{\mathrm{b}}(t)$, this yields the following explicit expression for the action $S$,

$$
\begin{aligned}
S=\int_{0}^{t_{0}} \mathrm{~d} t[ & \sum_{i} \frac{m_{i} a^{2}}{2}\left(\frac{\mathrm{d} \boldsymbol{x}_{i}}{\mathrm{~d} t}\right)^{2}+\frac{G}{a} \sum_{i \neq j} \frac{m_{i} m_{j}}{\left|\boldsymbol{x}_{i}-\boldsymbol{x}_{j}\right|} \\
& \left.+\frac{2}{3} \pi G \rho_{\mathrm{b}} a^{2} \sum m_{i} \boldsymbol{x}_{i}^{2}\right] .
\end{aligned}
$$

The exact equations of motion for the particles are then obtained from finding the stationary trajectories amongst the variations of the action $S$ subject to fixed boundary conditions at both the initial and final time.

Confining oneself to a feasible approximate evaluation in this Least Action Principle approach, one describes the orbits of particles, $\boldsymbol{x}_{i}(t)$, as a linear combination of suitably chosen universal functions of time with unknown coefficients specific to each particle presently located at a position $\boldsymbol{x}_{i, 0}$. For instance, by using the linear growth mode $D(t)$ as time variable (Giavalisco et al. 1993; Nusser \& Branchini 2000), one can parametrize the orbit $\boldsymbol{x}_{i}(D)$ of a particle as

$\boldsymbol{x}_{i}(D)=\boldsymbol{x}_{i, 0}+\sum_{n=1}^{N_{\mathrm{f}}} q_{n}(D) \boldsymbol{C}_{i, n}$

where the functions $q_{n}(D)$ form a set of $N_{\mathrm{f}}$ time-dependent basis functions. The factors $\boldsymbol{C}_{i, n}$ are then a set of free parameters, whose value is determined from evaluating the stationary variations of the action.

The functions $q_{n}(D)$ satisfy both two orbital constraints, necessary and sufficient to define solutions in agreement with evolution in the context of the Gravitational Instability theory for the formation of structure in the Universe: $q_{n}(1)=0$ ensures that at the present time the galaxies are located at their observed positions $\boldsymbol{x}_{i}(1)=\boldsymbol{x}_{i, 0}$ and $\lim _{D \rightarrow 0} D^{3 / 2} q_{n}(D) \theta(D)=0$ guarantees vanishing peculiar velocities at early epochs which, in turns, ensures initial homogeneity.

\subsection{Fast action minimization}

The successful application of the Least Action Principle towards probing the kinematics and dynamics of an evolving cosmological system depends to a large extent on the specific implementation. This will be dictated by the characteristics of the physical system. In order to enable a meaningful LAP analysis of large samples of galaxies, like the Local Universe samples studied in this work, an optimized procedure is necessary for dealing with the large number of objects. Nusser \& Branchini (2000) developed an optimized version of Peebles' original LAP formalism, the Fast Action Minimization method. The various optimization aspects of the FAM implementation proved to be crucial for our purposes. The relevant optimization hinges on four major aspects of the FAM scheme.

The first FAM improvement involves the choice of time basis functions $q_{n}(D)$. Its convenient choice of time basis functions yields a simple expression for the action of the system and for its derivatives with respect to $\boldsymbol{C}_{i, n}$. Both quantities relate to the internal gravity term $\boldsymbol{g}_{\text {int }}$ of the system. Once the action and its derivatives are evaluated numerically, the minimum of the action is determined by means of the conjugate gradient method (Press et al. 1992). The orbits of the system are then fully specified through the set of parameters $\boldsymbol{C}_{i, n}$ found in correspondence to the minimum.

Closely related to the first aspect is that of tuning the choice of the time basis functions $q_{n}(D)$ such that only a limited number $N_{\mathrm{f}}$ of basis functions is needed to successfully parameterize the orbits of the system. This is in particular beneficial to the the physical configuration we are studying here, involving Megaparsec scale dynamics characterized by quasi-linear or mildly nonlinear motions.

Note that using the growth factor $D$ as time variable makes the equations of motions almost independent of the value of $\Omega_{\mathrm{m}}$ (Nusser \& Colberg 2000). As a consequence FAM orbits and peculiar velocities in a generic $\Omega_{\mathrm{m}}$ universe can be obtained by appropriate scaling those assuming a flat cosmology. 
A final major aspect of the FAM implementation involves the efficient computation of the internal (self-consistent) gravity $\boldsymbol{g}_{\text {int }}$ from the particle distribution in the sample. To this end, the gravitational forces acting on the particles at the different epochs are computed by means of the TREECODE technique (Bouchet \& Hernquist 1988). By proceeding in this fashion, the FAM method is able to reconstruct the orbits of $\approx 10^{4}-10^{5}$ mass tracing objects back in time. This makes FAM numerically fast enough to perform a large number of orbit reconstructions, essential for performing the intended statistical analysis presented in the following sections.

In this work we use $N_{\mathrm{f}}=6$ basis functions to parameterize the orbits, choosing a tolerance parameter $t o l=10^{-4}$ to search for the minimum of the action $S$ and setting a softening parameter of $0.27 h^{-1} \mathrm{Mpc}$ to smooth the gravitational force in the TREECODE. Orbit searching in dynamically relaxed systems is a difficult exercise since one has to choose among the many solutions found at the extrema of the action. However, since the purpose of FAM is to investigate large scale dynamics dominated by coherent flows rather than virial motions, our evaluations translates into an orbit search restricted to solutions which do not deviate too much from the Hubble flow i.e. to the simplest orbits that represents the minima of the action. Therefore, we set the initial guess for $\boldsymbol{C}_{i, n}$ according to linear theory prescription and search for the minimum of the action to avoid multiple solutions found a stationary points which typically describe passing orbits (Peebles 1994). We have checked that this choice of parameters is optimal in the sense that decreasing tol, increasing $N_{\mathrm{f}}$ or changing the input set of $\boldsymbol{C}_{i, n}$ does not modify the final results appreciably.

Distortions in the resulting FAM-predicted peculiar velocities mainly arise from two systematic artifacts (Branchini et al. 2002). One is the discrete sampling of the mass distribution within $V_{\text {int }}$. The second, and overriding one, is the failure of FAM in reproducing the virial motions within high-density regions that is a direct consequence of having considered solutions that represents perturbations to the Hubble flow. This deficiency of the FAM reconstructions is clearly illustrated by the residual velocity vector maps (see Eq. (20)) in Figs. 7 and 8 (bottom row). These show the velocity vector differences between the "real" measured, i.e. $N$-body, velocities and the corresponding FAM reconstructions (here based on either the galaxy distribution in a $30 h^{-1} \mathrm{Mpc}$ central region or the extended $100 h^{-1} \mathrm{Mpc}$ region). The maps show how the largest residuals are the ones found in the high density regions: although the $\mathrm{FAM}_{30}$ and $\mathrm{FAM}_{100}$ velocity fields do show pronounced velocities near these regions they are not the proper "real" virialized velocities they should have been. The residual fields thus underline the fact that FAM's inaptitude to deal with regions characterized by large virial motions. Instead, in those situations it will lead to a false prediction of coherent inward streaming velocities, an effect pointed out by Nusser \& Branchini (2000) and which can be also noted in our images when carefully studying them.

Finally, for practical reasons, since we are merely interested in measuring the effect of external gravity fields we make a further simplifying hypotheses. We ignore redshift distortion effects by working in real space In this respect, we should point out that extensions of the action principle method allowing a direct processing of redshift space information have been proposed and shown to work (Phelps 2000; Phelps 2002; also see Sharpe et al. 2001).

\subsection{The role of biasing}

In this work we perform orbit reconstructions by assuming that all the mass of the systems is associated to point mass objects. More explicitly, we are making two different hypotheses. The first one is that we are able to identify a set of objects that trace the underlying mass density field in an unbiased way. The second one is that the internal structure of these objects is irrelevant for our reconstruction purposes.

The first assumption hardly applies to real galaxies that are most likely to be biased tracers of the mass distribution, as indicated by the relative bias between galaxies with different luminosities, colors and morphological type (Loveday et al. 1995). However, if galaxies and mass particles share the same velocity field so that the biasing relation remains constant along the streamlines, then the problem can be easily circumvented by specifying the biasing scheme at the present epoch (Nusser \& Branchini 2000).

Within the standard lore of galaxies embedded in a virialized halo of dark matter that grow through hierarchical merging of smaller systems, neglecting the internal structure of objects is an assumption that is best justified a posteriori by showing how well Numerical Action methods can reproduce $\mathrm{N}$-body velocities. Although the goodness of this assumption has been quantified by a number of numerical tests (e.g. Nusser \& Branchini 2000; and Branchini Eldar \& Nusser 2002) little effort has been devoted to understand why numerical action methods can accurately reconstruct the velocity field of a large $\mathrm{N}$-body simulation.

One of the reason for this success is that only $\sim 5 \%$ of the points used in our reconstructions, that were randomly selected from the $N$-body simulation, belong to virialized regions where FAM reconstruction fails. Fortunately, the locality of this "virial effect" allows us to partially circumvent this problem by applying a modest spherical tophat smoothing of $2 h^{-1} \mathrm{Mpc}$ to the FAM-predicted velocities. This tophat filter has been specifically important for the quantitative aspects of our study, where such systematic problems may sort distorting conclusions. This smoothing has been invoked in quantitative comparisons between FAM and $N$-body velocities presented in this work, in particular in the regression analyses.

Little is known about the ability of numerical action methods to reconstruct the orbits of virialized systems. Indeed, when applied to extended objects rather than point masses, numerical action methods follow a single center of mass point per virialized objects, completely neglecting its merging history. Some argument can be given to back our choice of neglecting the internal structure of virialized objects. First of all, after tracing back the merging history of virialized halos in $N$-body experiments a simple visual inspection reveals that particles ending up in the same halo at $z=0$ are contained within regions with simple boundaries at high redshifts. As a consequence, 


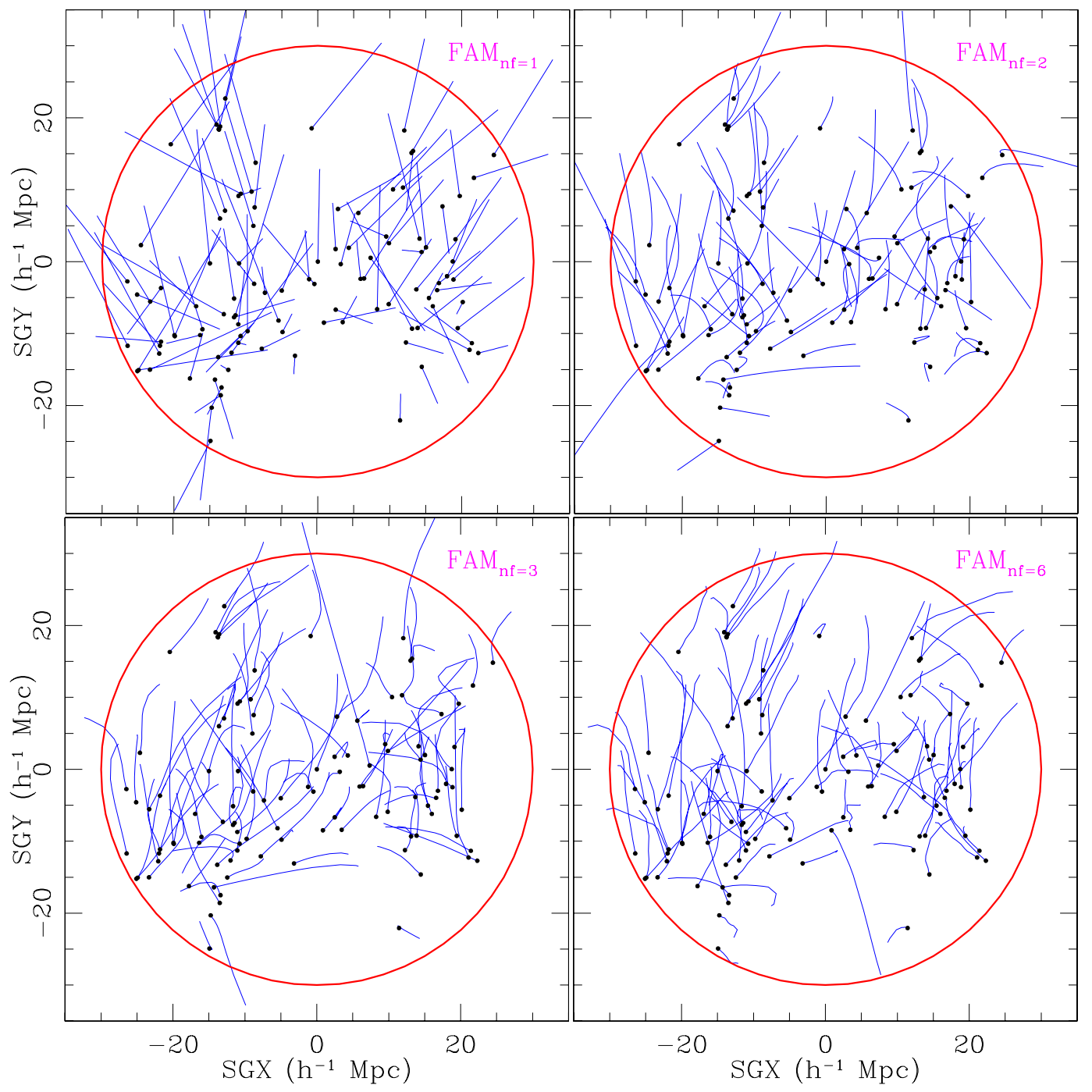

Fig. 1. 2D projected reconstructed FAM orbits for different values of $N_{\mathrm{f}}$. The black dots represent the final (present) positions for each object. The solid lines indicate the trajectories followed by the objects as a function of time. The top-left panel shows FAM reconstructed orbits with $N_{\mathrm{f}}=1$ (Zel'dovich approximation). The top-right with $N_{\mathrm{f}}=2$, the lower-left $N_{\mathrm{f}}=3$ and the lower-right $N_{\mathrm{f}}=6$. high order terms in the gravity potential about the halo center of mass are probably rather small. This probably minimize the role of major mergers whose rate for galaxy-size halos peaks in the redshift range 2-4 (Volonteri et al. 2003) while peculiar motions mostly develop at $z<2$ (Branchini \& Carlberg 1994). These qualitative arguments clearly need to be confirmed by appropriate numerical analyses similar to that of Branchini \& Carlberg (1994) but extending out to scales of cosmological interest.

\subsection{Ordered reconstructions}

To obtain an idea of the level of improvement obtained through the use of successively higher order FAM evaluations, Fig. 1 depicts 2D projections of the corresponding FAM particle orbit reconstructions within a local spherical volume of $\approx 30 \mathrm{~h}^{-1} \mathrm{Mpc}$. The black dots indicate the positions for each object in the sample, while the lines emanating from each dot represent the computed trajectories followed by these objects as they moved towards their present location. The illustrated configuration is taken from one of constructed mock catalogs, and resembles that of the Local Universe (see Sect. 5.2.1). Each successive FAM reconstruction is based on the same (present-day) particle distribution. The four frames correspond to successively higher order FAM approximations, involving an increasing number $N_{\mathrm{f}}$ of basis functions $q_{n}(D)$. The topleft panel shows FAM reconstructed orbits with $N_{\mathrm{f}}=1$, which in fact corresponds to the conventional first order Zel'dovich approximation and thus represent the orbits that would have been obtained by the PIZA method (Croft \& Gaztañaga 1997). These are followed by panels with $N_{\mathrm{f}}=2$ (top right), $N_{\mathrm{f}}=3$ (bottom left) and $N_{\mathrm{f}}=6$ (bottom right). They show a clear and steady improvement towards the $N_{\mathrm{f}}=6$ FAM evaluation. Testing proved that even higher order computations did not yield improvements significant enough to warrant the extra computational effort.

In summary, the galaxy orbits in our Local Universe environment are found at a minimum of the action which is not too far, yet different, from linear theory predictions. The FAM technique thus yields a significant modification of the recovered galaxy orbits and peculiar velocities for configurations that evolved well beyond the linear regime (see e.g. Fig. 1). Potentially its ability to deal with nonlinear circumstances might even prove of benefit to recover sets of cosmological initial conditions satisfying nonlinear observational 
constraints at the present day, which indeed has been suggested by Goldberg \& Spergel (2000).

\subsection{LAP and external forces}

The original cosmological Least Action Principle formulation by Peebles (1989) considered a fully self-consistent, i.e. isolated, system of point masses. For practical reasons, the original implementation had to be restricted to systems of at most a few dozen objects. Almost exclusively, the Local Group of galaxies formed the focus of these LAP studies (Peebles 1989, 1990, 1994; Dunn \& Laflamme 1993).

While these studies did indeed yield a substantial amount of new insight into the dynamical evolution of the Local Group, the issue of incorporating the dynamical influence exerted by external mass concentrations remained a major unsettled question. External forces do represent a significant component of the dynamics of the Local Group, as had been shown by Raychaudhury \& Lynden-Bell (1989). They established beyond doubt that the Local Group cannot be considered a tidally isolated entity, and demonstrated that the Local Group is acted upon by an appreciable quadrupolar tidal force. The resulting tidal torque appears to be responsible for the large angular momentum of the Local Group as a whole, as Dunn \& Laflamme (1993) showed in an elegant and pioneering analysis using orbits computed by the LAP variational method. They confirmed that the tidal influence of the external matter distribution is indeed essential to explain its present angular momentum.

In the course of time various strategies emerged to include external dynamical influences. The nature of these methods are mainly set by the character of the physical system under consideration, and to some extent was dependent on the available computational resources. Three strategies are outlined below.

\subsubsection{Directly including external masses}

To incorporate the external tidal influence within the LAP analysis the work by Peebles (1989, 1990, 1994), Peebles et al. (2001) and Dunn \& Laflamme (1993) attempted to identify a few principal external mass concentrations which would be responsible for the major share of the external gravitational influence. While in his first LAP study Peebles (1989) considered the Local Group mainly as an isolated system, sequel studies (Peebles 1990; Peebles et al. 2001) attempted to assess the possible external influence by neighbouring matter concentrations. In Peebles (1990) he attempted to condense the external tidal force into two nearby mass concentrations, the Sculptor and Maffei group, each modeled as a single mass. Both were incorporated as 2 extra particles, with properly scaled masses, within the action $S$ in order to take them along in a fully selfconsistent variational evaluation. A similar approach was followed by Dunn \& Laflamme (1993), be it that they included five galaxies/groups in the local cosmic neighbourhood which arguably contribute a significant torque on the Local Group. Also in a later application (Peebles et al. 2001) this approach was followed, be it with an extensive outer region between
$4 h_{75}^{-1} \mathrm{Mpc}$ and $20 h_{75}^{-1} \mathrm{Mpc}$ whose mass distribution was condensed into a coarse sample of some 14 major external objects.

This "self-consistent" strategy is feasible to pursue within the context of the original, computationally intensive, LAP implementation. This approach may therefore be followed in LG resembling situations in which a few objects suffice to represent the main aspects of a system's dynamical evolution. On the other hand, cosmic systems of a considerably larger scale than the Local Group would in general be too demanding for. Supercluster sized regions, with scales of up to a few tens of Megaparsec, count many more individual objects than a galaxy group. These systems have also not yet reached a formation stage so advanced that they have already largely decoupled from the global Hubble expansion, so the resulting external gravitational influence is usually the shared responsibility of a large number of external matter concentrations. Accounting for the large-scale tidal field would quickly become prohibitively expensive in terms of the computational effort for conventional LAP analyses.

\subsubsection{Inserting external tidal potential}

An alternative strategy is to incorporate the external gravity in the LAP scheme via an approximate expression for the external contribution. This may be most directly achieved by inserting an extra external tidal potential term $\Phi_{\text {tidal }}(t)$ in the action $S$ (Eq. (6)). As on sufficiently large, Megaparsec, scales we may expect this term to evolve according to linear gravitational instability perturbation growth,

$\Phi_{\text {tidal }}(t)=D_{\phi}(t) \Phi_{\text {tidal }}\left(t_{0}\right)=\frac{D(t)}{a(t)} \Phi_{\text {tidal }}\left(t_{0}\right)$,

in which $\Phi_{\text {tidal }}\left(t_{0}\right)$ is the tidal term at the present cosmic epoch and $D_{\phi}=(D / a)$ the linear growth term for the gravitational potential (the growth factor $D_{\phi}$ and cosmic expansion factor $a(t)$ are set to be equal to unity at the present epoch, $D\left(t_{0}\right)=$ $a\left(t_{0}\right)=1$.). Thus, instead of evolving it self-consistently along with the considered system, the external field is determined at one epoch - usually the present one - and then incorporated as an extra linearly growing gravity field term $\Phi_{\text {tidal }}(t)$ in the action $S$ :

$$
\begin{aligned}
S=\int_{0}^{t_{0}} \mathrm{~d} t[ & \sum_{i} \frac{m_{i} a^{2}}{2}\left(\frac{\mathrm{d} \boldsymbol{x}_{i}}{\mathrm{~d} t}\right)^{2}+\frac{G}{a} \sum_{i \neq j} \frac{m_{i} m_{j}}{\left|\boldsymbol{x}_{i}-\boldsymbol{x}_{j}\right|} \\
& \left.+\frac{2}{3} \pi G \rho_{\mathrm{b}} a^{2} \sum m_{i} \boldsymbol{x}_{i}^{2}-\Phi_{\text {tidal }}(t)\right]
\end{aligned}
$$

There are various possibilities to compute the tidal potential term $\Phi_{\text {tidal }}$, usually from the current mass distribution. One option is to compute it directly from a sample of $M_{\text {ext }}$ external objects which is deemed responsible and representative for the major share of the external tidal force field,

$\Phi_{\text {tidal }}\left(t_{0}\right)=\sum_{i} m_{i}\left\{\frac{G}{a} \sum_{j=1}^{M_{\text {ext }}} \frac{m_{j}}{\left|\boldsymbol{x}_{i}-\mathbf{y}_{j}\right|}\right\}$.

Note that none of these external objects $\left(j=1, \cdots, M_{\text {ext }}\right)$ is taken into account as far as the action of the system and the 
computation of their orbits is concerned, except for their "passive" role in determining $\Phi_{\text {tidal }}$. An alternative approach is to insert an approximate analytical expression for $\Phi_{\text {tidal }}$, in particular one including the dipolar and quadrupolar contributions, $\boldsymbol{d}$ and $\boldsymbol{T}$, to the tidal potential,

$\Phi_{\text {tidal }}\left(t_{0}\right)=\sum_{i} \boldsymbol{d} \cdot \boldsymbol{x}_{i}+\frac{1}{2} \boldsymbol{x}_{i} \cdot \boldsymbol{T} \cdot \boldsymbol{x}_{i}$.

Equivalently, one may chose to insert the corresponding expressions directly into the expression for the derivative of the action with respect to an expansion coefficient, $\partial S / \partial C_{i, n}^{\alpha}=0$, evidently equal to zero within this variational approach.

The first, "direct", procedure (Eq. (11)) was followed by Shaya et al. (1995), who for the purpose of studying the velocity field within the surrounding $30 \mathrm{~h}^{-1} \mathrm{Mpc}$ modelled the relevant external mass distribution after the distribution of rich Abell clusters from Lauer \& Postman (1994). To some extent, Sharpe et al. (2001) operated along the same lines, be it that they added the resulting tidal term directly to the reconstructed velocities produced by the LAP procedure. However, while in principle exact, such a concentrated and static mass distribution may involve considerable uncertainties and can be highly sensitive to the uncertainties in the location of a few dominant point masses. As this spatial point distribution is supposed to form a suitable model for the underlying large scale matter distribution this may be even more worrisome.

Potentially more elegant may therefore be the modelling of a smooth tidal field along the line of the second procedure (Eq. (12)), as suggested by Schmoldt \& Saha (1998). The corresponding dipolar and quadrupolar term may then be based on the best available determinations of these parameters. On the other hand, when the LAP volume is comparatively large, the analytical approximation may represent an oversimplification of the force field, neglecting potentially important local variations within the external force field.

\subsubsection{Selfconsistent and direct FAM approach}

The indirect "potential" approach which we described above (Eqs. (11) or (12)) may not properly account for the temporal evolution of the external field in the case of nonlinearly evolving systems. The formalism assumes a static, merely linearly evolving, gravitational potential. However, the matter concentrations which generate the external tidal forces will themselves get displaced as the cosmos evolves. These displacements may be relatively minor for distant masses, but for the more nearby entities this may be entirely different. A detailed treatment of the external mass distribution will be necessary when the influence of the nearby external objects on the evolution of small "interior" regions is comparable to or even dominant over the selfgravity of the region. It will be equally crucial to follow the detailed whereabouts of nearby matter concentrations in the case of a large "interior" region in which a marked contrast between the central regions and the outer realms may result in a significantly different dynamic evolution.

This prompted us to follow an alternative and direct approach, a fully self-consistent strategy in which also the
Table 1. $N$-body simulation parameters. Column 1: cosmological model. Column 2: $\Omega_{0}$ mass density parameter. Column 3: $\Lambda$, cosmological constant parameter. Column $4: \Gamma$, power spectrum shape parameter. Column 5: $\sigma_{8}$, density perturbation amplitude spectral normalization. Column 6: size of the computational box in $h^{-1} \mathrm{Mpc}$. Column 7: number of particles in the simulations.

\begin{tabular}{lcccccc}
\hline \hline Cosmology & $\Omega_{0}$ & $\Lambda$ & $\Gamma$ & $\sigma_{8}$ & Boxlength & $N_{\text {obj }}$ \\
\hline$\Lambda \mathrm{CDM}$ & 0.3 & 0.7 & 0.25 & 1.13 & 345.6 & $192^{3}$ \\
$\tau \mathrm{CDM}$ & 1.0 & 0.0 & 0.25 & 0.55 & 345.6 & $192^{3}$ \\
\hline
\end{tabular}

external matter concentrations are accounted for in the computation of the system of evolving particle orbits. Alongside that in the "local" region for which we seek to reconstruct the velocity field, also the system of objects in the exterior regions (30 $h^{-1} \mathrm{Mpc}<r<100 h^{-1} \mathrm{Mpc}$ ) are considered. Non-uniform manifestations of the external influence can only be included by pursuing such a direct and systematic approach. It is only through the availability of the FAM technology that we were enabled to do so for a Megaparsec system consisting of a large number of objects.

\section{Cosmological scenarios}

The mock catalogs on which we apply our Fast Action Minimization analysis are extracted from $N$-body simulations in two different cosmological settings. Their characteristics, in terms of their relevant parameters, are listed in Table 1. The table also lists the simulation specifications. The first scenario is a flat $\Lambda$ CDM model with a cosmological constant term $\Omega_{\Lambda, 0}=0.7\left(\Omega_{0}=0.3, \Omega_{0}+\Omega_{\Lambda, 0}=1.0, \Gamma=0.25, n=1\right)$. The second model is a $\tau$ CDM Einstein-de Sitter $\left(\Omega_{0}=1.0, \Omega_{\Lambda, 0}=\right.$ $0, \Gamma=0.25, n=1)$ model, motivated by the decaying particle model proposed by Bond \& Efstathiou (1991). Both scenarios were chosen to be viable with respect to the current observational constraints, implying similarities in many overall properties and appearances, yet with some significant differences with respect to their dynamical repercussions. This may provide indications on whether the galaxy motions in our local cosmic neigbourhood do contain information on the structure formation scenario.

In both cases the amplitude of density fluctuations is normalized on the basis of the observed abundance of rich galaxy clusters in the local universe. This abundance depends on the magnitude of the matter field fluctuations on the mass scale characteristic for galaxy clusters. This translates into a dependence on the amplitude of density fluctuations on cluster scales modulated by the mean global matter density. A variety of studies (e.g. White et al. 1993; also see Eke et al. 1996) found that in order to yield the present-day cluster abundance the amplitude of density fluctuations in spheres of radius $8 \mathrm{~h}^{-1} \mathrm{Mpc}, \sigma_{8}$, and $\Omega_{0}$ are related by

$\sigma_{8}=0.55 \Omega_{0}^{-0.6}$.

The resulting power spectra are depicted in Fig. 2 (top left). On all scales, the density fluctuations in the $\tau \mathrm{CDM}$ scenario, 

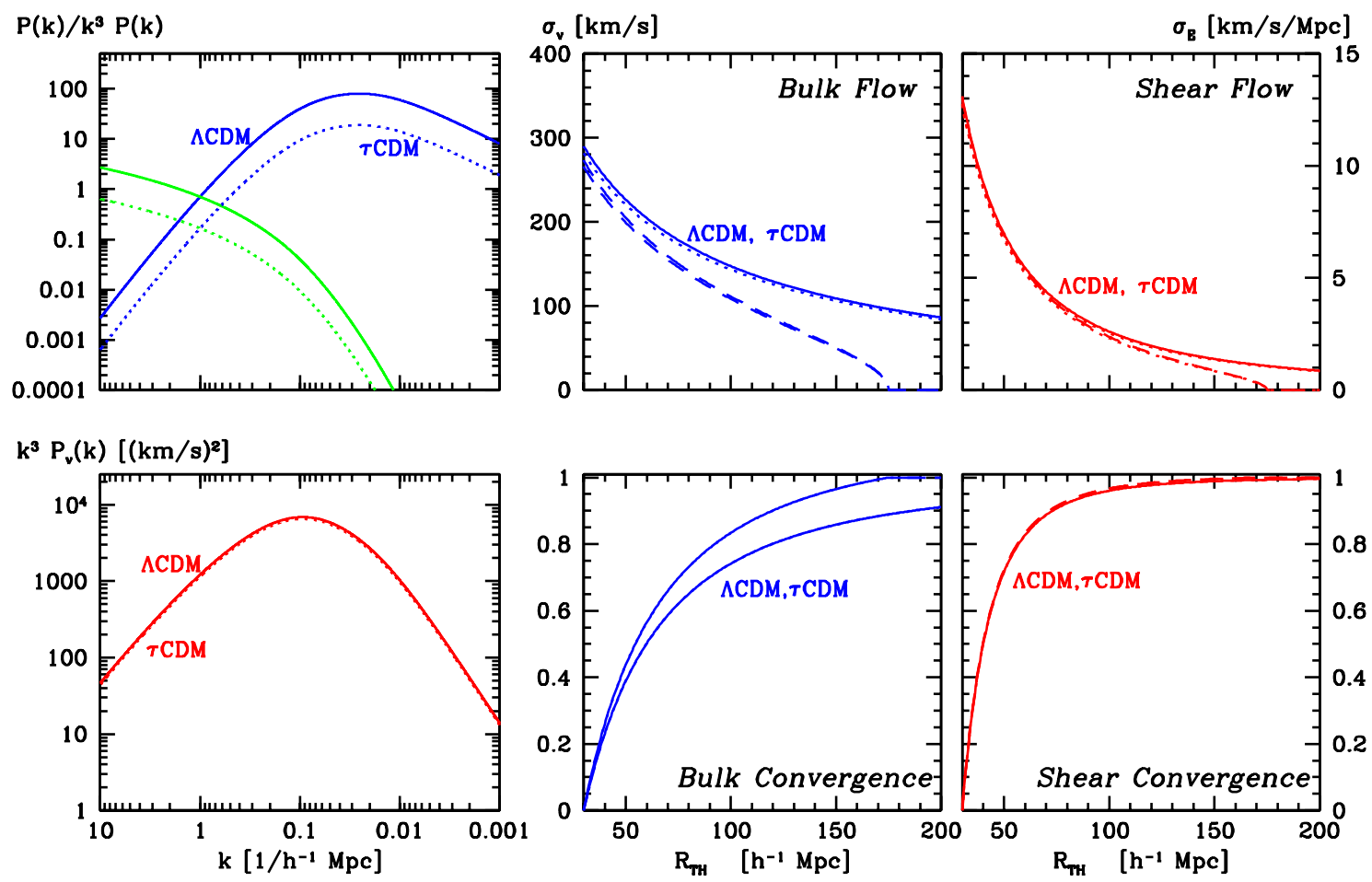

Fig. 2. Spectral characteristics for the two studied cosmological scenarios. Upper left: power spectrum $P(k)$ (green lines) and $k^{3} P(k)(b l u e ~ l i n e s)$, for the $\Lambda \mathrm{CDM}$ (continuous lines) and $\tau \mathrm{CDM}$ (dotted lines) cosmological scenarios used in this study. Lower left: velocity power $k^{3} P_{\mathrm{v}}(k)$, the amount of velocity power per logarithmic waveband interval. Upper centre: expected bulk flow velocities ( $\mathrm{km} \mathrm{s}^{-1}$ ) over a spherical (tophat) volume of radius $R_{\mathrm{TH}}$. The solid line is the real theoretical prediction, the dashed one concerns the restricted power spectrum represented in the simulation box. Centre low: the convergence of the bulk flow, defined according to Eq. (16). Upper right: expected shear flow (km s${ }^{-1} \mathrm{Mpc}^{-1}$ ) over a spherical (tophat) volume of radius $R_{\mathrm{TH}}$. Similar to bulk flow, the solid line concerns the full theoretical power spectrum while the dashed corresponds to the restricted power spectrum represented in the simulation box. Lower right: convergence of the shear flow, defined according to Eq. (16). Note that for the righthand frames the corresponding ordinate axis are the ones on the righthand side of the plotbox, while for the central frames it is the one on its lefthand side.

represented by the dotted lines (for both $P(k)$ (green lines) and $k^{3} P(k)$ (blue lines)), are less pronounced than those of the $\Lambda \mathrm{CDM}$ scenario: the two power spectra have a similar shape and differ by a simple scaling factor over the entire wavelength range. Visually, this is immediately reflected in the stark differences between the spatial galaxy distribution in the resulting mock catalogs. Figure 3 provides such a visual comparison. It shows the "external" PSC $z$ catalog mimicking galaxy distribution in three mutually perpendicular central slices in the case of the $\Lambda \mathrm{CDM}$ scenario (top row), together with the same set of frames for a $\tau \mathrm{CDM}$ mock galaxy catalog (bottom row). On all scales, the $\tau \mathrm{CDM}$ galaxy distribution looks considerably more uniform than that in the $\Lambda \mathrm{CDM}$ Universe. Not only is the clustering of galaxies in the $\Lambda \mathrm{CDM}$ scenario more pronounced, it also delineates considerably larger structures, a manifestation of the power spectrum's amplitude at the corresponding large wavelengths.

Because the higher average matter density in the $\Omega_{0}=1$ $\tau \mathrm{CDM}$ Universe does almost fully compensate for the lower amplitude of the density fluctuations the resulting gravity and velocity perturbation fields in the $\Lambda \mathrm{CDM}$ and $\tau \mathrm{CDM}$ scenarios are very similar. The velocity power spectra $k^{3} P_{\mathrm{v}}(k)$ are shown in the bottom lefthand panel of Fig. 2: their functional dependence is the same over the entire wavelength range. The larger mass corresponding to a given density excess in the $\tau \mathrm{CDM}$
Universe evidently effects a stronger gravitational force. The resulting large scale motions scale as $f\left(\Omega_{0}\right) \propto \Omega_{0}^{0.6}$. This happens to be almost exactly the inverse of the average density perturbation amplitude scaling (Eq. (13)), which is proportional to $\Omega_{0}^{-0.6}$ (Eq. (13)). While this is exactly the factor involved in the normalization of the power spectrum, in terms of $\sigma_{8}$, the lower level of density fluctuations gets precisely compensated by the higher amount of mass involved with them. This can be directly observed from the velocity power spectra $P_{\mathrm{v}}(k)$ for the two scenarios (Fig. 2, lower lefthand frame). The velocity power spectra for both scenarios are exactly equal over the entire wavelength range, both in functional dependence as well as in amplitude. Note that also the gravity perturbations in the $\Lambda \mathrm{CDM}$ scenario are substantially stronger than those in the $\tau$ CDM cosmology: because they scale with $\frac{3}{2} \Omega H_{0}^{2}$ and the amplitude of the density perturbations, which according to Eq. (13) is $\propto \Omega^{0.6}$, the average peculiar gravitational acceleration is proportional to $\Omega^{0.4} H_{0}^{2}$.

The comparison between $k^{3} P(k)$ (Fig. 2, top panel, blue lines) and $k^{3} P_{\mathrm{v}}(k)$ (Fig. 2, bottom panel) in the same figure shows the shift of the velocity perturbations, with respect to the density perturbations, towards a more large-scale dominated behaviour. This follows directly from the continuity equation, connecting the velocity and density perturbations such that 
the velocity power spectrum relates to $P(k)$ through $P_{\mathrm{v}}(k) \propto$ $P(k) k^{-2}$.

The large-scale behaviour of the (linear) velocity perturbation field immediately illuminates the difficulty in tracing the full array of matter inhomogeneities responsible for the cosmic motions within a specific cosmic region. To account for all noticeable contributions it is necessary to probe out to large depth. This is manifestly evident for the first order component in the externally induced flow, the "bulk flow" $v_{\text {bulk. }}$. A measure for the expected bulk flow within a (tophat) spherical region of size $R_{\mathrm{TH}}$,

$\boldsymbol{v}_{\text {bulk }}(\boldsymbol{x}) \equiv \int_{V} \mathrm{~d} \boldsymbol{x}^{\prime} \boldsymbol{v}\left(\boldsymbol{x}^{\prime}\right) W_{\mathrm{TH}}\left(\boldsymbol{x}-\boldsymbol{x}^{\prime}, R_{\mathrm{TH}}\right)$,

is represented by the (root square) average value $\sigma_{\mathrm{v}}$, whose value may be inferred from the Fourier integral

$$
\begin{aligned}
\sigma_{\mathrm{v}}\left(R_{\mathrm{TH}}\right) & =H_{0} f\left(\Omega_{0}\right) \sigma_{-1} \\
& \equiv H_{0} f\left(\Omega_{0}\right) \sqrt{\int \frac{\mathrm{d} \boldsymbol{k}}{(2 \pi)^{3}} P(\boldsymbol{k}) \hat{W}_{\mathrm{TH}}^{2}(\boldsymbol{k}) k^{-2}} .
\end{aligned}
$$

In these relations, $W_{\mathrm{TH}}\left(\boldsymbol{x}, R_{\mathrm{TH}}\right)$ and $\hat{W}_{\mathrm{TH}}(\boldsymbol{k})$ are the expressions for the tophat window filter, spatially and in Fourier space, and $\sigma_{-1}$ is the spectral moment $\sigma_{j}$ for $j=-1$ (see Bardeen et al. 1986, henceforth BBKS).

How substantial the large scale origin of the bulk flow is may be readily appreciated from Fig. 2 (top centre). Because the linear character of fluctuations on large scales, the spectral $\sigma_{\mathrm{v}}$ (Eq. (16)) does provide a reasonable order-of-magnitude estimate of the magnitude of the large-scale bulk motions. The figure shows the estimated bulk flow amplitudes, $\approx \sigma_{\mathrm{v}}$, as a function of the (tophat) window radius: the bulk flow is clearly a large scale phenomenon, converging only very slowly towards large spatial scales. In both the $\Lambda \mathrm{CDM}$ scenario and the $\tau \mathrm{CDM}$ scenario the externally induced bulk flow on a scale of $30 \mathrm{~h}^{-1} \mathrm{Mpc}$ will be in the order of $200 \mathrm{~km} \mathrm{~s}^{-1}$. Of this overall bulk flow, more than $100 \mathrm{~km} \mathrm{~s}^{-1}$ has to be ascribed to inhomogeneities on scales exceeding $200 h^{-1} \mathrm{Mpc}$ ! When assessing the motions in a local volume of $30 \mathrm{~h}^{-1} \mathrm{Mpc}$ radius, in terms of relative external contributions, inhomogeneities on a scale larger than $100 h^{-1} \mathrm{Mpc}$ still contribute more than $25 \%$ of the total while the ones larger than $200 h^{-1} \mathrm{Mpc}$ are still responsible for more than $10 \%$ (see Fig. 2, centre bottom). We should therefore expect to find substantial external contributions in the $\Lambda \mathrm{CDM}$ and $\tau \mathrm{CDM}$ simulations. Note that this relative contribution to the bulk flow, the "bulk convergence", is defined as the relative contribution by matter perturbations within a radius $R_{\mathrm{TH}}$ to the externally induced bulk flow on a scale of $30 h^{-1} \mathrm{Mpc}$ (the size of the NBG volume):

$\mathcal{F}_{\text {bulk }} \equiv 1-\frac{\sigma_{\mathrm{v}}\left(R_{\mathrm{TH}}\right)}{\sigma_{\mathrm{v}}\left(30 h^{-1} \mathrm{Mpc}\right)}$.

The second order aspect of the velocity field which we seek to study is the induced velocity shear $s_{i j}$,

$s_{i j} \equiv \frac{1}{2}\left\{\frac{\partial v_{i}}{\partial x_{j}}+\frac{\partial v_{j}}{\partial x_{i}}\right\}-\frac{1}{3}(\nabla \cdot \boldsymbol{v}) \delta_{i j}$
Also the velocity shear reveals interesting and distinguishing differences between the $\Lambda \mathrm{CDM}$ and the $\tau \mathrm{CDM}$ scenario. In the linear regime the expected magnitude of the shear tensor $s_{i j}$, on a tophat scale $R_{\mathrm{TH}}$, may be evaluated through its direct proportionality to the tidal shear $\mathcal{T}_{i j}$. Quantifying $s_{i j}$ by means of its (root square) average $\sigma_{\mathrm{s}}$ (van de Weygaert \& Bertschinger 1996), we find

$\sigma_{\mathrm{s}}=H_{0} f\left(\Omega_{0}\right) \sigma_{0}\left(R_{\mathrm{TH}}\right) \sqrt{\frac{1-\gamma^{2}}{15}}$

in which the (dimensionless) spectral parameter $\gamma \equiv \sigma_{1}^{2} / \sigma_{0} \sigma_{2}$ is defined through the 0th, 1st and 2nd spectral moments $\sigma_{j}$ (see BBKS 1986). The predictions for the two cosmological scenarios are shown in topright frame of Fig. 2. With respect to the bulk flow there is a marked difference in coherence scale: the major contributors to the tidal shear are located at considerably closer distances than the sources of the bulk flow (Fig. 2, cf. lower right with lower centre). Most of the shear inducing matter inhomogeneities are found within a radius of $100 \mathrm{~h}^{-1} \mathrm{Mpc}$, accounting for more than $95 \%$ of its value (Fig. 2, lower right). On a scale of $30 \mathrm{~h}^{-1} \mathrm{Mpc}$ we expect an external tidal shear of $\approx 7 \mathrm{~km} \mathrm{~s}^{-1} \mathrm{Mpc}^{-1}$ for both the $\Lambda \mathrm{CDM}$ scenario and the $\tau \mathrm{CDM}$ model.

\section{Bulk flow and tidal shear: velocity flow multipole components}

In the previous sections we have found that in order to obtain a good representation of the local cosmic velocity field it is necessary to take into account the external gravitational influence. This was accomplished through the incorporation of the fully detailed external mass distribution contained in the (fluxlimited) galaxy catalogs. This involved the galaxy distribution out to distances of $100 h^{-1} \mathrm{Mpc}$. The reconstructions showed that modelling of velocity fields by FAM with the inclusion of matter concentrations on such large scales is indeed rewarding.

In nearly all situations where the local volume $V_{\text {int }}$ is suitably large, the small-scale details of the external mass configuration are rather irrelevant for constructing an appropriate model of the flows in the local Universe. An appropriate approximate expression for the the gravitational potential $\Phi_{\text {ext }}(\boldsymbol{r})$ inside the internal volume $V_{\text {int }}$ due to the surrounding external matter distribution follows from its expansion in multipole contributions. Assuming a spherical local volume with radius $R_{\text {int }}$, the potential $\Phi_{\text {ext }}$ may be written in terms of a multipole expansion of spherical harmonics $Y_{l m}(\theta, \phi)$ (see e.g. Jackson 1975)

$$
\begin{aligned}
\Phi_{\text {ext }}(\boldsymbol{r}) & =-\int_{R_{\text {int }}}^{\infty} \frac{G \rho\left(\boldsymbol{x}^{\prime}\right)}{\left|\boldsymbol{x}-\boldsymbol{x}^{\prime}\right|} \mathrm{d} \boldsymbol{x}^{\prime} \\
& =-\sum_{l=0}^{\infty} \sum_{m=-l}^{m=l} \frac{4 \pi G}{2 l+1} Q_{l m} Y_{l m}(\theta, \phi) r^{l},
\end{aligned}
$$

in which the multipole moments $Q_{l m}$ relate to the external density field $\rho\left(\boldsymbol{x}^{\prime}\right)$ as

$Q_{l m}=\int_{r}^{\infty} \rho\left(\boldsymbol{x}^{\prime}\right) r^{\prime l-3} Y_{l m}^{*}\left(\theta^{\prime}, \phi^{\prime}\right) \mathrm{d} \boldsymbol{x}^{\prime}$ 
Most contributions to the external gravity $\boldsymbol{g}_{\text {ext }}$ will be confined to these dipole and quadrupole components, induced by the corresponding large-scale constellations in which the surrounding matter concentrations have grouped themselves. Here we will assess the approximation in which the potential expansion (Eq. (23)) is restricted to the monopole term $l=0$, the dipole term $l=1$ and the quadrupole term $l=2$,

$\Phi_{\mathrm{ext}}(\boldsymbol{r}) \approx \Phi_{0}(\boldsymbol{r})+\Phi_{1}(\boldsymbol{r})+\Phi_{2}(\boldsymbol{r})$

with

$\Phi_{l}(\boldsymbol{r})=-\sum_{m=-l}^{m=l} \frac{4 \pi G}{2 l+1} Q_{l m} Y_{l m}(\theta, \phi) r^{l}$.

To explore the nature of the external component in the total gravitational field in the Local Universe we proceed by probing it through the resulting peculiar velocity field. The amplitude of higher order terms may be assumed to be so small that one cannot expect to deduce any significant value, given the sizeable errors in the available galaxy peculiar velocity datasets, we may expect this to be a reasonable approximation. We investigate the velocity field by decomposing the residual velocity field - i.e. the component in the velocity field which could not be accounted for in the $\mathrm{FAM}_{30}$ reconstruction and supposedly induced by external influences - into its multipole components. Once we have determined the bulk flow component and shear tensor components in the tidal velocity field, we will assess whether we can indeed relate this to the external gravitational ("tidal") influence within the local Universe.

Restricting the description of the external gravitational influence to the first few orders of its multipole expansion has several advantages. The large-scale external dipole and quadrupole gravity perturbations retain a largely linear character, simplifying the velocity field analysis and thus retaining the direct linear relation between gravity and velocity field. Also, by discarding its small-scale fluctuating contributions a physically more transparent image of the velocity field is obtained. This allows a straightforward relation and translation towards the corresponding large-scale pattern of the surrounding mass distribution. A final practical issue of some importance is the fact that the dipole and quadrupole characterization is particularly suited for an implementation in FAM. Restricting the external force field to these moments alleviates the need to take into account a large sample of external galaxies. Not only is the latter computationally expensive, in practice it is even not always feasible.

\subsection{Velocity field multipole decomposition}

In the multipole analysis we restrict ourselves to the externally induced velocity components, $\boldsymbol{v}_{\text {ext }}$, which in the following we frequently designate by the term "tidal"1. For each object, the "tidal" velocity vector is determined by subtracting the internally induced velocity field, $\boldsymbol{v}_{\text {int }}$, from the object's full velocity.

\footnotetext{
${ }^{1}$ In the following we regularly use the word "tidal" to shortly indicate the externally induced component of a gravity or velocity field. As it includes a dipolar contribution, strictly speaking this is not an appropriate term. Also see Sect. 3.6.2.
}

The latter is usually the $N$-body velocity of the mock galaxy, although we will assess the possibility of using the FAM 100 velocity as a reasonable alternative. The internal velocity $\boldsymbol{v}_{\text {int }}$ is deduced by evaluating, through our FAM computations, the impact of the internal matter distribution within the internal catalog volume $V_{\text {int }}$. The resulting (residual) peculiar velocity vector $\boldsymbol{v}_{\text {ext }}$ field may then be expressed in terms of a Taylor series description as function of spatial position $\boldsymbol{x}$.

For the practical implementation, we follow the general scheme described by Kaiser (1991). The velocity field Taylor expansion is truncated at the quadratic term and is restricted to the dipole and quadrupole moments (and a minor monopole term). The tidal velocity field $\boldsymbol{v}_{\text {ext }}$, is then modeled by the the first two components, a bulk flow vector, $\tilde{u}_{i}$, and a quadratic shear tensor contribution, $\tilde{s}_{i j}$,

$v_{\mathrm{ext}, i}=\tilde{u}_{i}+\tilde{s}_{i j} x \hat{x}_{j}, \quad$ where $\quad i, j=\{1,2,3\}$,

in which $i, j$ denotes the Cartesian component indices. As in Eq. (21), the vectors $\hat{x}_{j}$ represent the vector components along the Cartesian $j$ direction of the spatial unity vector oriented along the object position vector $\boldsymbol{x}$. Using these notations, we can easily reconfigure Eq. (26) and express the $i$-component of the velocity of object $n$ into a product of the vectors $F_{n, I}$ and $V_{I i}$,

$v_{n, i}=\sum_{I=1}^{4} F_{n, I}(\boldsymbol{x}) V_{I i}$

in which the data 4-vector $F_{I}$ and the velocity field component 4-vector $V_{I i}$ are defined as

$F_{n, I}=\left\{1, x \hat{x}_{1}, x \hat{x}_{2}, x \hat{x}_{3}\right\}$

$V_{I i}=\left\{\tilde{u}_{i}, \tilde{s}_{i 1}, \tilde{s}_{i 2}, \tilde{s}_{i 3}\right\}$.

The "dipolar" bulk flow components $\tilde{u}_{i}$ and "quadrupolar" velocity shear components $\tilde{s}_{i j}$ can then be obtained by solving for the vectors $V_{I i}$ on the basis of a fitting analysis (to be precise, $\tilde{s}_{i j}$ also includes a minor residual "monopole" expansion/contraction term). We accomplish this by computing for each Cartesian component $i$ the values for the multipole elements $\tilde{u}_{i}$ and $\tilde{s}_{i j}$ which minimize $\chi^{2}$

$\chi^{2}=\sum_{n=1}^{N_{\mathrm{objs}}}\left(v_{n, \mathrm{ext}, i}-\sum_{I} F_{n, I}(\boldsymbol{x}) V_{I i}\right)^{2}$,

to be evaluated on the basis of the data sample of $N_{\text {obj }}$ objects at locations $\boldsymbol{x}_{n}$ and with inferred "external" velocities $\boldsymbol{v}_{n \text {,ext }}$. The bulk flow and velocity shear in the externally induced velocity component $\boldsymbol{v}_{\text {ext }}$, along with a residual expansion term,

$v_{\mathrm{ext}, i} \approx v_{\mathrm{exp}, i}+v_{\mathrm{bulk}, i}+v_{\mathrm{shear}, i}$

will follow directly from the inferred values of the 4-vector components $V_{I i}$ :

$$
\begin{array}{ll}
v_{\text {exp }, i}=\frac{1}{3} \operatorname{Tr}(\tilde{s}) x \hat{x}_{i} & \\
v_{\text {bulk }, i}=\tilde{u}_{i} ; & v_{\text {shear }, i}=\sum_{j=1}^{3} \tilde{s}_{i j}^{\prime} x \hat{x}_{j}
\end{array}
$$


Table 4. Average results for the tidal bulk amplitude and shear eigenvalues. The errors represent the $1 \sigma$ scatter around the average value. Column 1: tidal field region where the velocity components were computed. The first two sections refer to the tidal field computed from the $N$-body - FAM models. In the third section the external tidal influences are determined by the large region modeled on FAM $_{100}$ instead of the $N$-body samples. The fourth region refers to the residual field between $N$-body and $\mathrm{FAM}_{\mathrm{mpl}}$ after the external contributions have been accounted for in the FAM modeling: FAM $_{30}+$ tidal bulk + tidal shear (see text Sects. 8.2 and 8.5, for details). Column 2: cosmological model. Column 3: bulk flow, $v_{\text {bulk }}$. Column 4: shear eigenvalue amplitude, $|s|$. Column 5: $s_{1}$ eigenvalue (stretching). Column 6: $s_{2}$ eigenvalue (middle). Column 7: $s_{3}$ eigenvalue (compressional).

\begin{tabular}{|c|c|c|c|c|c|c|c|}
\hline $\begin{array}{l}\text { Field } \\
\text { configuration }\end{array}$ & Cosmology & $\begin{array}{c}v_{\text {bulk }} \\
\left(\mathrm{km} \mathrm{s}^{-1}\right)\end{array}$ & $s$ & $s_{1}$ & $s_{2}$ & $s_{3}$ & \\
\hline & $\Lambda \mathrm{CDM}$ & $313 \pm 65$ & $13.3 \pm 3.8$ & $8.8 \pm 3.6$ & $0.6 \pm 2.5$ & $-9.4 \pm 2.3$ & $\left(\mathrm{~km} \mathrm{~s}^{-1} \mathrm{Mpc}^{-1}\right)$ \\
\hline & $\tau \mathrm{CDM}$ & $216 \pm 57$ & $13.6 \pm 4.6$ & $8.9 \pm 3.1$ & $1.1 \pm 2.0$ & $-10.0 \pm 3.7$ & \\
\hline \multicolumn{8}{|c|}{$N$-body $-\mathrm{FAM}_{30}$} \\
\hline & $\Lambda \mathrm{CDM}$ & & $399 \pm 115$ & $264 \pm 107$ & $19 \pm 76$ & $-283 \pm 70$ & $\left(\mathrm{~km} \mathrm{~s}^{-1}\right)$ \\
\hline & $\tau \mathrm{CDM}$ & & $409 \pm 137$ & $267 \pm 92$ & $33 \pm 60$ & $-300 \pm 110$ & \\
\hline & $\Lambda \mathrm{CDM}$ & $81 \pm 51$ & $3.7 \pm 1.2$ & $2.6 \pm 0.9$ & $-0.2 \pm 0.8$ & $-2.4 \pm 1.0$ & $\left(\mathrm{~km} \mathrm{~s}^{-1} \mathrm{Mpc}^{-1}\right)$ \\
\hline & $\tau \mathrm{CDM}$ & $121 \pm 54$ & $4.8 \pm 1.1$ & $3.4 \pm 1.0$ & $0.0 \pm 0.8$ & $-3.3 \pm 0.8$ & \\
\hline \multicolumn{8}{|c|}{$N$-body $-\mathrm{FAM}_{100}$} \\
\hline & $\Lambda \mathrm{CDM}$ & & $110 \pm 36$ & $78 \pm 27$ & $-6 \pm 25$ & $-72 \pm 30$ & $\left(\mathrm{~km} \mathrm{~s}^{-1}\right)$ \\
\hline & $\tau \mathrm{CDM}$ & & $145 \pm 33$ & $101 \pm 30$ & $-1 \pm 23$ & $-100 \pm 23$ & \\
\hline & $\Lambda \mathrm{CDM}$ & $317 \pm 89$ & $14.0 \pm 4.6$ & $9.4 \pm 4.0$ & $0.5 \pm 2.9$ & $-9.9 \pm 3.0$ & $\left(\mathrm{~km} \mathrm{~s}^{-1} \mathrm{Mpc}^{-1}\right)$ \\
\hline & $\tau \mathrm{CDM}$ & $245 \pm 60$ & $15.3 \pm 4.3$ & $9.8 \pm 2.5$ & $1.4 \pm 2.9$ & $-11.2 \pm 3.8$ & \\
\hline \multicolumn{8}{|c|}{$\mathrm{FAM}_{100}-\mathrm{FAM}_{30}$} \\
\hline & $\Lambda \mathrm{CDM}$ & & $421 \pm 138$ & $281 \pm 120$ & $15 \pm 86$ & $-296 \pm 91$ & $\left(\mathrm{~km} \mathrm{~s}^{-1}\right)$ \\
\hline & $\tau \mathrm{CDM}$ & & $458 \pm 128$ & $293 \pm 75$ & $42 \pm 87$ & $-335 \pm 115$ & \\
\hline & $\Lambda \mathrm{CDM}$ & $81 \pm 51$ & $2.4 \pm 1.3$ & $-0.6 \pm 1.7$ & $-0.2 \pm 1.4$ & $0.9 \pm 1.3$ & $\left(\mathrm{~km} \mathrm{~s}^{-1} \mathrm{Mpc}^{-1}\right)$ \\
\hline \multicolumn{8}{|c|}{$N$-body $-\mathrm{FAM}_{\mathrm{mpl}}$} \\
\hline & $\tau \mathrm{CDM}$ & $121 \pm 54$ & $2.9 \pm 0.8$ & $-0.6 \pm 1.6$ & $-0.5 \pm 1.6$ & $0.9 \pm 1.7$ & \\
\hline
\end{tabular}

in which $\operatorname{Tr}(s)$ is the trace of the tensor $s_{i j}$ and $\tilde{s}_{i j}^{\prime}$ the traceless shear tensor

$$
\begin{aligned}
\operatorname{Tr}(\tilde{s}) & \equiv \tilde{s}_{11}+\tilde{s}_{22}+\tilde{s}_{33} \\
\tilde{s}_{i j}^{\prime} & =\tilde{s}_{i j}-\frac{1}{3} \operatorname{Tr}(\tilde{s}) \delta_{i j} .
\end{aligned}
$$

\subsection{Velocity multipole analysis: results}

The results of our analysis are summarized in Table 4. It lists the average quantities for the tidal bulk flow and shear components for the two cosmological scenarios discussed in this work. The table has been organized in four (horizontal) sections. Each corresponds to another "differential" velocity field, the difference between two differently processed velocity fields.

For both the $\Lambda \mathrm{CDM}$ and the $\tau \mathrm{CDM}$ model each of the quoted values in Table 4 involve the average and standard deviation determined on the basis of ten different realizations. This adds up to 8 configurations, two cosmologies per section. For each of the 8 configurations, in the third column the table lists the dipole component of the external velocity field, the bulk flow $v_{\text {bulk. }}$. Subsequently, the velocity shear is specified in terms of the three eigenvalues $s_{1}, s_{2}$ and $s_{3}$ of the traceless shear tensor. This is preceded in the fourth column by the amplitude $s$ of the shear. Note that shear is quoted in two units. First, units of $\mathrm{km} \mathrm{s}^{-1} \mathrm{Mpc}^{-1}$, followed by the equivalent velocity differential in $\mathrm{km} \mathrm{s}^{-1}$ over a volume of $30 \mathrm{~h}^{-1} \mathrm{Mpc}$ radius. The intention of the latter is to offer a directly appreciable comparison between the relative importance of bulk flow and shear contributions.

Each of the four sections specifies the values of the computed dipole and quadrupole moments of the velocity field of the corresponding sample. The first section relates to a multipole analysis of the differential velocity field between the full $N$-body velocity field and the $\mathrm{FAM}_{30}$ velocity reconstructions of the inner $30 h^{-1} \mathrm{Mpc}$ region, $N$-body $-\mathrm{FAM}_{30}$. The resulting residual velocity field has been generated by the mass distribution beyond a radius of $30 \mathrm{~h}^{-1} \mathrm{Mpc}$. On these linear scales the inferred dipole and quadrupole components of the velocity field may be directly related to the moments of the surrounding mass distribution.

The second section of Table 4 does the same for the larger $100 \mathrm{~h}^{-1} \mathrm{Mpc}$ region. The outcome of similar analyses are presented in the third and fourth section. The third section repeats the analysis of the first section, except that the external tidal influences are determined on the basis of the difference between the FAM velocity reconstructions within the large $100 h^{-1} \mathrm{Mpc}$ region and the inner $30 \mathrm{~h}^{-1} \mathrm{Mpc}$ region. Earlier, in Sect. 6.3, we have found that the major share of the origin of the external tidal field is confined to this region and that it therefore may 
well be determined from the residuals between FAM $_{100}$ and $\mathrm{FAM}_{30}$. The comparison between the inferred multipole moments of the velocity differences between FAM $100-\mathrm{FAM}_{30}$ in the third section and those in the first section are therefore expected to be rather similar, any systematic differences originating in tidal effects generated beyond a radius of $100 h^{-1} \mathrm{Mpc}$. The fourth section in Table 4 refers to the values of the residual tidal velocity field between $N$-body $-\mathrm{FAM}_{\mathrm{mpl}}$, the FAM sample after having accounted for the missing external tidal contributions, $\mathrm{FAM}_{30}+$ tidal bulk + tidal shear (see Sect. 8.5). If indeed all significant contributions can be characterized by their dipolar and quadrupolar contributions, the multipole values in this section are expected to be negligible.

\subsection{Velocity multipole contributions: maps}

For a direct visual appreciation of the various multipole contributions to the tidal velocity field we assess the "tidal" velocity field $N$-body $-\mathrm{FAM}_{30}$, the velocity field generated by the mass distribution beyond a radius of $30 h^{-1} \mathrm{Mpc}$, for one of the $\Lambda \mathrm{CDM}$ catalogs. The presented maps concern the same $\Lambda \mathrm{CDM}$ catalog as those presented in the maps of Figs. 7. The map of the projection of this "tidal" velocity flow onto three central planes is shown in the top row of Fig. 13.

\subsubsection{Dipolar component: bulk flow}

The externally generated velocity flow is dominated by its bulk flow component. This is in general true for both cosmologies. The large impact of the bulk flow over the local $30 h^{-1} \mathrm{Mpc}$ volume can be immediately inferred from the values in the first section of Table 4, revealing contributions in excess of $200 \mathrm{~km} \mathrm{~s}^{-1}$.

To facilitate visual appreciation of this observation we have have reoriented the reference system in Fig. 13 such that the $x$-axis is oriented along the bulk flow. While the original Cartesian system is an arbitrary one and thus lacks a physical context, the "bulk flow reference system" confines the inferred bulk flow $\tilde{u}$ exclusively to the $x$-direction. As a result there are no bulk flow components in the corresponding $y$ - and $z$-direction (note that within the $y-z$ plane their direction is arbitrarily defined). The pre-eminence of the bulk flow component can be immediately seen in the $x-y$ and $x-z$ frames in the top row of Fig. 13. Note that the same velocity maps, mostly so the $y-z$ frame, reveal a clear shear pattern.

\subsubsection{Quadrupolar component: velocity shear}

Seeking to assess the quadrupolar term in the external velocity field we first remove the remaining expansion term from $\tilde{s}_{i j}$. Diagonalization of the resulting traceless shear tensor $\tilde{s}_{i j}^{\prime}$ yields the shear eigenvalues and eigenvectors. The eigenvalues $s_{1}, s_{2}$ and $s_{3}$ are indicative for the strength of the tidal force field induced by the surrounding matter distribution, while the principal directions of this quadrupolar velocity perturbation field are indicated by the corresponding eigenvectors $\hat{e}_{s, i}$.
The "shear ellipsoid", the quadratic surface defined by the shear tensor $\tilde{s}_{i j}^{\prime}$ with principal axes aligned along the eigenvectors and with axis size set by the corresponding eigenvalue $s_{i}$, defines a natural reference system to assess the tidal shear flow field. The coordinate axes of this "shear reference frame" are identified with the orthonormal basis defined by the (normalized) eigenvectors. The $x$-axis is chosen to be aligned along the major axis of the "shear ellipsoid", the direction defined by the largest (positive) eigenvalue $s_{1}$ and directed along the strongest dilational (stretching) motion incited by the external tidal field. Likewise the $z$-axis is chosen to coincide with the lowest (negative) eigenvalue $s_{3}$, aligned along the strongest "compressional" component of the tidal velocity flow. This leaves the $y$-axis as the one coinciding with the intermediate eigenvalue $s_{2}$.

The imprint of the shearing motions can be discerned within the $y-z$ plane and, most prominently, along the " $x-z$ " projection of the "bulk flow reference system". After subtraction of the bulk flow component, i.e. $N$-body-FAM $30^{-} v_{\text {bulk }}$, the quadrupolar component of the externally induced velocity flow represents its principal constituent (Fig. 13, lower row). This is confirmed by the values quoted in Table 4 for the shear contribution. In particular when stated in the velocity equivalent unit of $\mathrm{km} \mathrm{s}^{-1}$ these shear values suggest that the quadrupolar shear contributions are of a comparable magnitude to those of the bulk flow. The maps in the lower row of Fig. 13 suggest that there are strong dilational and compressional motions within the $y-z$ plane. By contrast, the shear motions in the $x$ direction appear to be uncommonly weak. Given the "bulk flow reference system", it implies that for this particular realization we see a bulk flow directed almost perpendicular to the shear flow motions.

Figure 14 depicts the same $\Lambda \mathrm{CDM}$ mock sample as presented in Figs. 7, 8 and 13, here in the "shear reference frame". The top row shows the full externally induced flow field, $N$-body $-\mathrm{FAM}_{30}$, in this reference system. The tidal shear flows are almost exclusively confined to the $x-z$ plane. This is most evidently illustrated in the central row of frames showing the velocity field without its bulk flow component: hardly any systematic flow is noticeable in the $y$-direction of the intermediate shear eigenvalue.

\subsection{Multipole scale dependence}

When turning to the external influences over a large $100 h^{-1} \mathrm{Mpc}$ region, we may conclude from the second section of the table that most of the external contributions are accounted for, both bulk flow and shear are at least a factor of 3-4 smaller than for the inner $30 h^{-1} \mathrm{Mpc}$ region. The third and fourth section show that the explicit contributions from the regions between $100 h^{-1} \mathrm{Mpc}$ and $30 h^{-1} \mathrm{Mpc}$ and those beyond $100 h^{-1} \mathrm{Mpc}$ are indeed significantly different, those beyond $100 h^{-1} \mathrm{Mpc}$ tending towards zero contributions and as far as the shear is concerned almost an order of magnitude smaller than the equivalent contributions by the 30-100 $h^{-1} \mathrm{Mpc}$ region. 


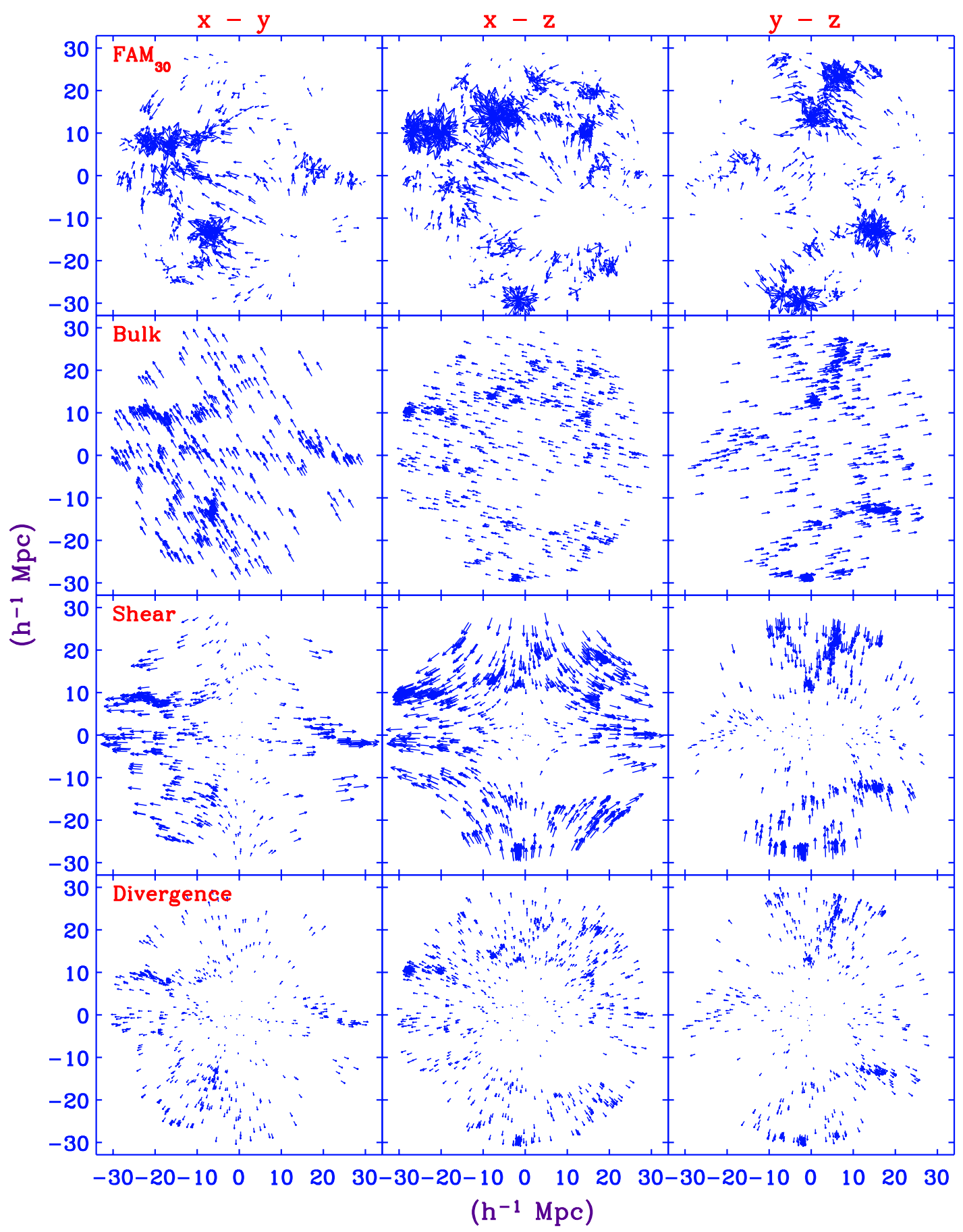

Fig. 12. Velocity Field Multipole Decomposition: the total $N$-body velocity field, involving the same one as in Figs. 13 and 14 , decomposed into its four different components. The coordinate system is that defined by the tidal shear tensor, see Fig. 13. At each row we depict the velocities in the $(x-y),(x-z)$ and $(y-z)$ plane. Top: the locally induced velocity, approximated by the FAM F $_{30}$ realization. Second row: the monopole component, a result of the slight local expansion due to its underdensity wrt. the global Universe. Third row: the bulk flow, of which most is concentrated in the $(x-y)$ and $(y-z)$ plane. Fourth row: the shear flow component.

A similar graphical assessment involving the $\mathrm{FAM}_{100}$ reconstructions emphasizes the minor significance of tidal contributions stemming from density fluctuations beyond a radius of $100 \mathrm{~h}^{-1} \mathrm{Mpc}$. No coherent velocity pattern can be recognized in the residual velocity field between full $N$-body and FAM 100 reconstruction. The comparison between this residual velocity field with the velocity maps including the contributions of the inferred bulk flow and shear flow do hardly show any difference. In all cases the velocity fields are dominated by the same thermal motions.

\subsection{Multipole velocity flow model}

Following our argument that the externally induced velocity flow within the inner $30 \mathrm{~h}^{-1} \mathrm{Mpc}$ mainly consists of a bulk flow 


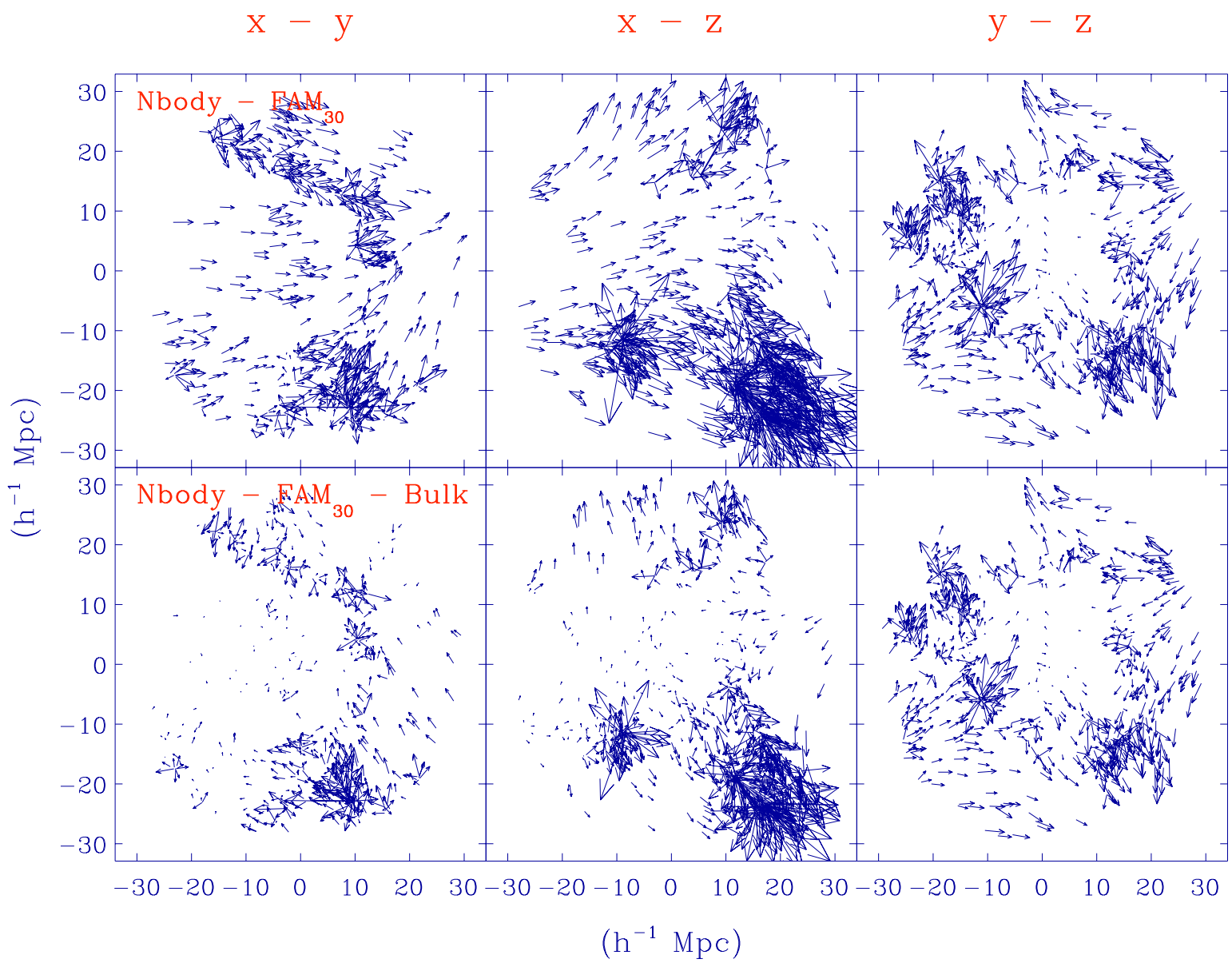

Fig. 13. 2D projected peculiar (residual) velocities for the same mock catalog as in Figs. 7 and 8 in three perpendicular central planes of $10 h^{-1} \mathrm{Mpc}$ width. The coordinate frame is rotated such that the bulk flow velocity is oriented along the $x$-direction. Within the plane perpendicular to the $x$-axis, the $y$ and $z$ axes are chosen arbitrarily. First row: the residual velocity (i.e. tidal velocity) $N$-body $-\mathrm{FAM}_{30}$. Second row: residual velocities after subtraction bulk flow component. The resulting residual field is clearly dominated by a shear pattern, most notably in the $y-z$ plane.

and shear contribution, we may expect that the effect of the external gravity field can be sufficiently accounted for by adding these components to a local velocity field model based on the mass distribution in and around the Local Superclusters.

By separating the "internal" FAM velocity field from the "external" multipole contributions of the (monopole,) dipole and quadrupole components of the "tidal" velocity field and adding the two, we obtain a total "FAM-multipole" model ve-

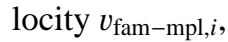

$v_{\mathrm{fam}-\mathrm{mpl}, i} \approx v_{\mathrm{FAM}, i}+v_{\mathrm{exp}, i}+v_{\mathrm{bulk}, i}+v_{\mathrm{shear}, i}$.

A visual impression of the extent of the successive multipole contributions may be obtained from Fig. 12. The vector plots of the four velocity contributions to $v_{\text {fam-mpl }}$ (Eq. (32)) are depicted in four successive rows, each within the mutually perpendicular three central slices (wrt. the shear reference system). The top row concerns the FAM 30 velocity field reconstruction, followed successively by the expansion/contraction term (monopole), the bulk flow (dipole) and velocity shear (quadrupolar).

From Figs. 13 and 14 we conclude that the differences between the "full" $N$-body velocities and $v_{\text {fam-mpl }}$, the total sum of the internal $\mathrm{FAM}_{30}$ and external dipole and quadrupole contributions, do not appear to show systematic trends as it can be noticed from the residual bulk and shear components in Sect. 4 of Table 4 . Wherever there are large deviations, these are mainly confined to the high density virialized regions.

\subsection{Point-to-point comparison}

A quantitative quality assessment of the "FAM-multipole" model is offered by the point-to-point comparison between the full $N$-body velocity and its difference with respect to the successive modes of the "FAM-multipole" velocity in Fig. 15. The $v_{x}, v_{y}$ and $v_{z}$ of the various velocity components refer to the "bulk flow reference system". The top row, plotting $v_{N \text { body }}$ vs. the residual $N$-body-FAM 30 , reveals the expected systematic differences due to missing externally induced contributions. Given the fact that the bulk flow in this reference system is confined to the $x$-component, we may note the uniform systematic shift of the $x$ residuals with respect to the zeropoint $\left(v_{N \text { body }}, v_{\text {res }}\right)=(0,0)$ (top lefthand frame). The subsequent addition of the dipolar bulk flow contribution to $\mathrm{FAM}_{30}$ leads to a systematic downward uniform vertical shift of 


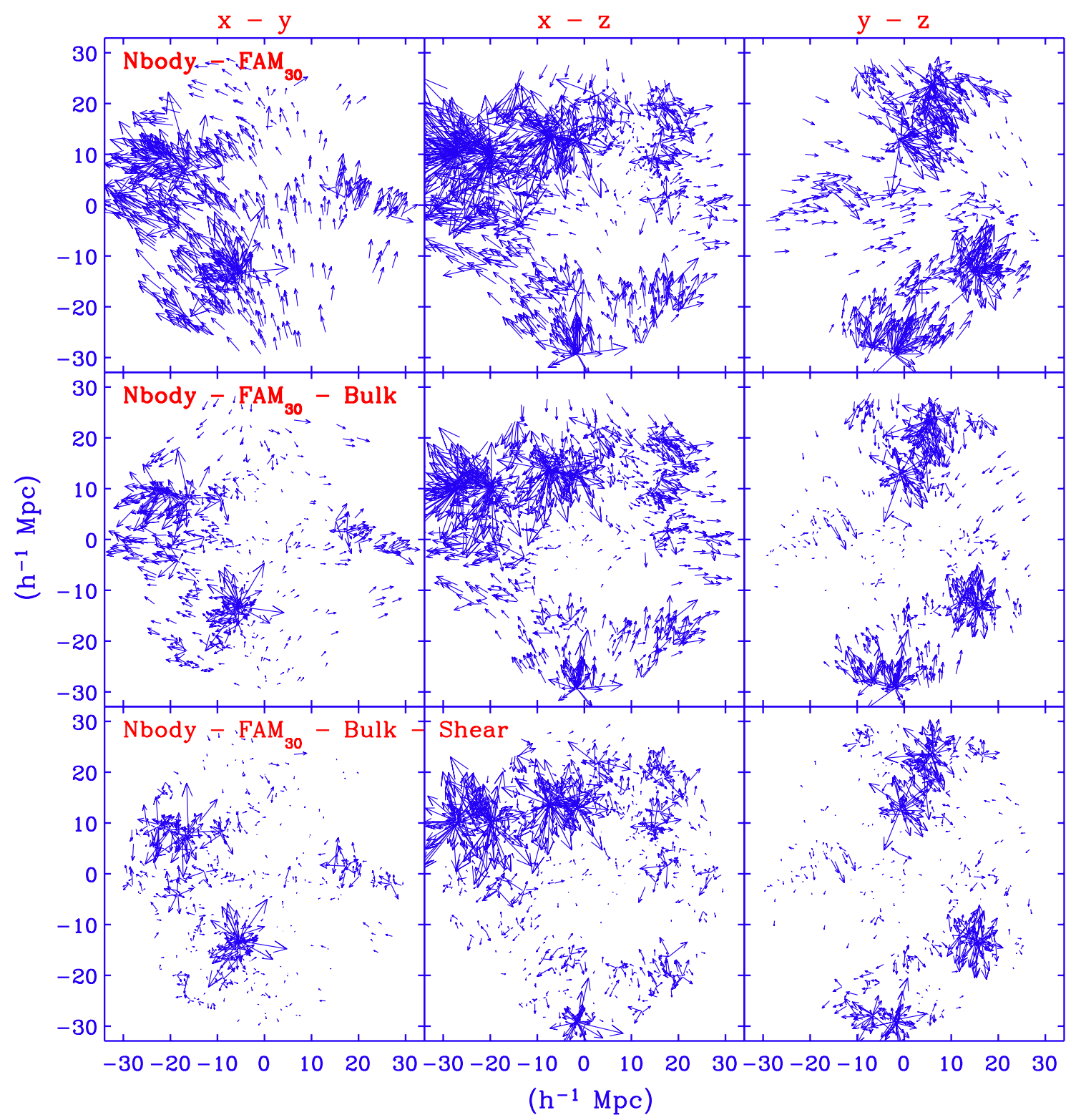

Fig. 14. 2D projected peculiar velocities for the same mock catalog as in Figs. 7 and 8 in three perpendicular central planes of $10 h^{-1}$ Mpc width. The coordinate frame is defined by the three eigenvectors of the shear tensor. The $x$-axis is aligned along the direction of the largest (stretching) eigenvalue, the $y$-axis is aligned along the middle eigenvalue and the $z$-axis along the smallest (compressional) eigenvalue. The first two rows are the same as Fig. 13, within the "shear reference system". The third row depicts the final residual field after subtraction of the quadrupolar shear component.

$N$-body-FAM 30 -Bulk (middle row Fig. 15): also the residuals in the $x$-direction now center on $v_{\text {res }}=0$ (note that by virtue of the bulk flow the $N$-body velocities in the $x$-direction are also skewed to values larger than $v_{N \text { body }}=0$ ).

The three point-to-point diagrams in the middle row of Fig. 15 show that even while the bulk flow is taken into account systematic motions remain in all three directions. The point-to-point comparisons still follow a strong correlation with respect to the the $N$-body velocities. It mainly involves the presence of the quadrupolar velocity shear component (in addition to a minor ingredient contributed by the monopole expansion/contraction term). This can be immediately inferred from the comparison between the diagrams in the central and lower row of Fig. 15: once the quadrupole component "Shear" has been added to the "FAM $30+$ Bulk" velocities the systematic effects seem to have largely vanished. What remains in the residuals is mainly random scatter, centered on the $v_{\text {res }}=0 \mathrm{~km} \mathrm{~s}^{-1}$ line, with some exceptional outliers originating in the virialized regions.

We have quantified the point-to-point comparisons by performing linear regressions similar to those presented in Sect. 7. Table 5 summarizes the results of this comparison for all catalog samples for both cosmologies. In both cosmological models the slope of the best fitting line is consistent with unity at $\sim 1 \sigma$ confidence level. As expected, the scatter around the fit is similar to that of all previous analyses (see Table 3). Offsets around the zero-point are consistent with zero, although with a large dispersion. The strength of the point-to-point 


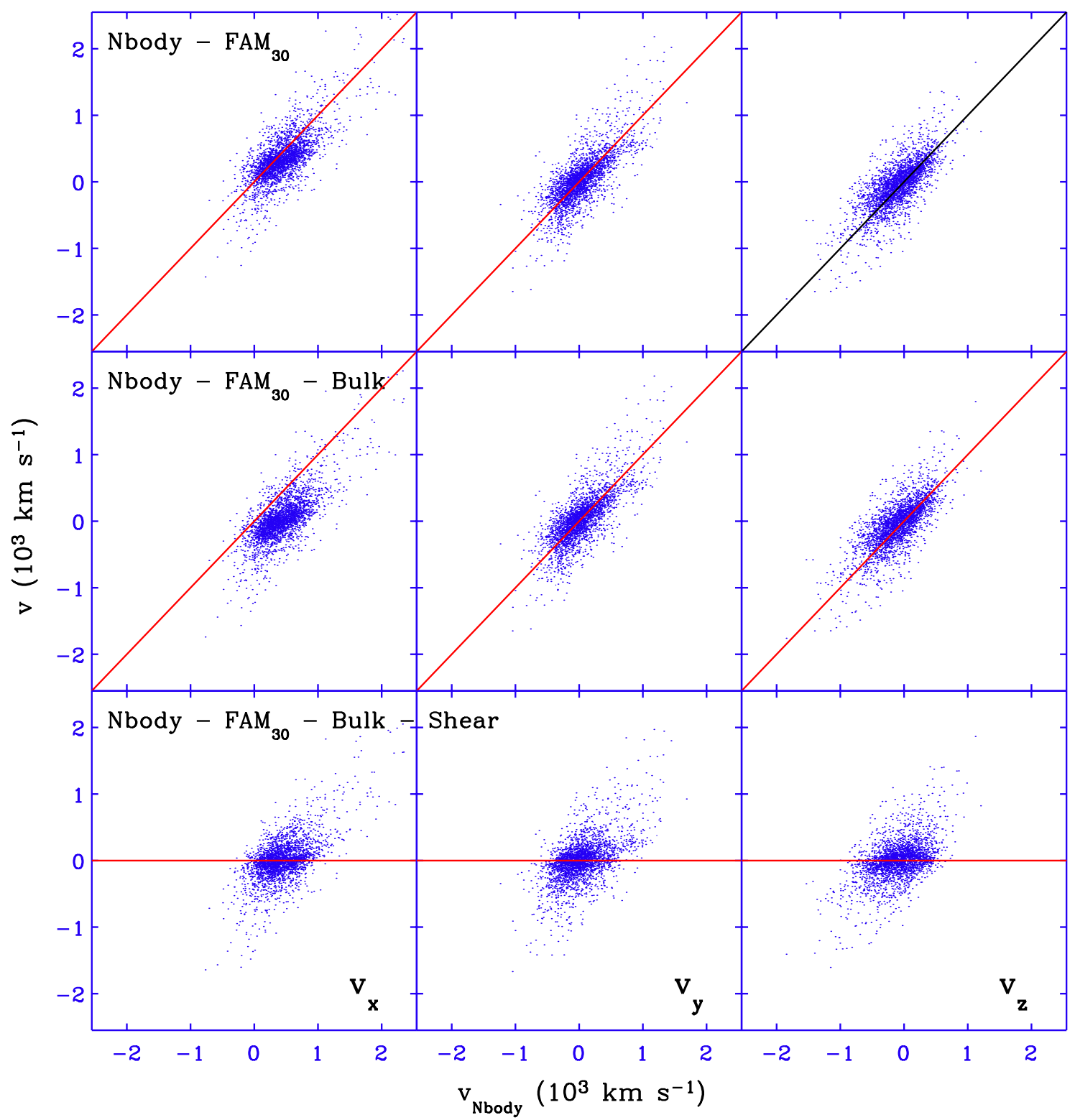

Fig. 15. point-to-point comparison between the three successive residual velocities and the corresponding mock catalog $N$-body velocity. The three panels in each row correspond to the $x, y$ and $z$ velocity components. The coordinate system is the "shear reference system". Top row: the tidal velocity field $N$-body-FAM. Middle row: residual velocity field after subtraction bulk flow. Bottom row: residual velocity field after subtraction of both tidal bulk and shear components.

correlations has increased considerably with respect to their FAM $_{30}$ counterpart (Table 3) and it is very similar to the $\mathrm{FAM}_{100}$ case.

\subsection{Surrounding matter distribution: tidal source}

The surrounding external matter distribution is the source for the tidal velocity field which we inferred in the previous sections. For various purposes we wish to relate the computed dipolar bulk flow and quadrupolar shear flow components to the surrounding matter distribution which induced them.

The induced tidal velocities involve spatial scales ranging from $30 h^{-1} \mathrm{Mpc}$ to $100 h^{-1} \mathrm{Mpc}$. Over this range the linear theory of gravitational instability holds to good approximation. This translates into a direct linear relationship between induced velocity $\boldsymbol{v}_{\text {ext }}$ and the cumulative external gravitational force $g_{\text {ext }}$,

$\boldsymbol{v}_{\mathrm{ext}}(\boldsymbol{x}, t)=\frac{2 f(\Omega, \Lambda)}{3 H \Omega} \boldsymbol{g}_{\mathrm{ext}}(\boldsymbol{x}, t)$,

with $f(\Omega, \Lambda)$ the linear velocity growth factor. This linear relationship also holds for every component of the velocity and gravity fields, and thus also for the individual dipolar and quadrupolar components of the externally induced velocity field. They are directly proportional to equivalent dipole and quadrupolar components of the gravity field:

$$
\begin{gathered}
\boldsymbol{v}_{\text {bulk }}(\boldsymbol{x}, t)=\frac{2 f(\Omega, \Lambda)}{3 H \Omega} \boldsymbol{g}_{\text {bulk }}(\boldsymbol{x}, t) \\
s_{i j}(\boldsymbol{x}, t)=-\frac{2 f(\Omega, \Lambda)}{3 H \Omega} \mathcal{T}_{i j}(\boldsymbol{x}, t)
\end{gathered}
$$


Table 5. Average final results for the $N$-body velocities vs. the corrected $\mathrm{FAM}_{30}$ after adding to it the tidal Bulk and Shear contributions. The errors represent the $1 \sigma$ scatter around the average value. Column 1: cosmological model. Column 2: non parametric (Spearman) correlation coefficient. Column 3: linear correlation index. Column 4: zero point of the best linear fit. Column 5: slope of the best fitting line. Column 6: dispersion around the fit.

\begin{tabular}{lccccc}
\hline \hline Cosmology & $R_{\text {Spear }}$ & $R_{\text {lin }}$ & $\begin{array}{c}a_{0} \\
\left(\mathrm{~km} \mathrm{~s}^{-1}\right)\end{array}$ & $a_{\text {lrg }}$ & $\begin{array}{c}\sigma_{\text {lrg }} \\
\left(\mathrm{km} \mathrm{s}^{-1}\right)\end{array}$ \\
\hline$\Lambda \mathrm{CDM}$ & $0.62 \pm 0.17$ & $0.58 \pm 0.22$ & $-4.03 \pm 75.45$ & $0.83 \pm 0.20$ & $246 \pm 134$ \\
$\tau \mathrm{CDM}$ & $0.75 \pm 0.11$ & $0.71 \pm 0.14$ & $-9.31 \pm 30.19$ & $0.93 \pm 0.13$ & $219 \pm 86$ \\
\hline
\end{tabular}

with the (external) gravitational tidal shear tensor $\mathcal{T}_{i j}$ is defined as (see van de Weygaert \& Bertschinger 1996),

$\mathcal{T}_{i j}=\frac{\partial^{2} \Phi_{\text {tidal }}}{\partial x_{i} \partial x_{j}}$

Notice that because of its external nature, the term $\frac{1}{3} \nabla^{2} \Phi_{\text {tidal }} \delta_{i j}$ is always equal to zero.

Ideally, we would like to infer the external tidal potential $\Phi_{\text {tidal }}$ directly from the galaxy distribution in a sufficiently large surrounding region. This is specifically true for its dipolar and quadrupolar moments, with the intention to insert these terms directly into the expression for the FAM potential (Eqs. (12) and (10)). The required externally induced bulk flow velocity and velocity shear should be the result. The comparison of the FAM computed velocities for the local volume, in combination with the computed tidal velocities (Sect. 8.2), and the observed and measured velocities would then enable us to determine the amount of mass and average density in the local volume.

To determine the gravitational influence of the surrounding matter distribution, we set out to assess the sky distribution of galaxies in the local NBG volume, out to $r_{\mathrm{NBG}}=30 h^{-1} \mathrm{Mpc}$, along with the external mass distribution in radial shells out to a distance $r_{\mathrm{PSC} z} \leq 100 \mathrm{~h}^{-1} \mathrm{Mpc}$. A prominent dipolar matter configuration in the sky distribution will translate into a strong bulk gravity force. Similarly, quadrupolar anisotropies will translate into an effective tidal shear force. In Fig. 16 we have plotted the galaxies in one of our $\Lambda \mathrm{CDM}$ mock catalogs in five successive distance shells. Aitoff projections of the angular positions of the galaxies, as seen from the centre of the local NBG volume, provide an impression of the level of anisotropy in the mass distribution at successive radii.

The first sky plot (top sphere) depicts the sky position of the galaxies in the local NBG-mimicking mock sample. It involves a highly flattened distribution, perhaps reminiscent of the Supergalactic Plane. The four subsequent shells correspond to successive cuts through the $\mathrm{PSC} z$ mimicking samples, at sampling depths $d_{\text {sur }}=$ [0-30], [30-55], [55-70] and [85-100] $h^{-1} \mathrm{Mpc}$. The first and direct observation is the diminishing sample density as a function of survey depth, in accordance with the selection function (Eq. (19)). Structure is most prominent in the first shell, at $d_{\text {sur }}=[30-55] h^{-1} \mathrm{Mpc}$ (central left sphere). The structure contained in this shell also shows a clear affiliation with the matter distribution in the local NBG volume. The compact massive concentration at $l \approx 220^{\circ}$ is clearly connected to a dense region in the local "plane". A superficial inspection of the angular galaxy distribution reveals the presence of strong dipolar and quadrupolar components, effecting considerable tidal forces. Note that both external shells display a rather strong concentration of galaxies in their southern hemisphere, in the vicinity of $l \approx 180-200^{\circ}$. Similar but weaker contributions can also be recognized from the galaxy distribution in the shell between $d_{\text {sur }} \approx 70-85 h^{-1} \mathrm{Mpc}$. Beyond $d_{\text {sur }}>85 h^{-1} \mathrm{Mpc}$, however, the angular pattern appear to be considerably less pronounced. This is in line with the earlier findings that there were hardly noticeable tidal contributions from large distances.

To see to what extent the depicted galaxy distribution can indeed be held responsible for most of the inferred tidal bulk flow and tidal shear, we have determined the corresponding bulk force $\boldsymbol{g}_{\text {bulk }}$ (Eq. (4)) and tidal shear $\mathcal{T}_{i j}$ (Eq. (5)) evoked by the external galaxy distribution $\left(r>30 h^{-1} \mathrm{Mpc}\right)$. Since we do not have a continuous density field but the positions of a finite number of objects in our galaxy flux-limited and full mass distribution catalogs, the bulk acceleration on the LG is computed from the discrete equivalent. For a sample of galaxies at locations $\boldsymbol{x}_{i}$, with an average number density $n$ of selected objects, this leads to

$\boldsymbol{g}_{\text {bulk }}=\frac{H f(\Omega, \Lambda)}{4 \pi n} \sum_{i} \frac{1}{\psi\left(x_{k}\right)} \frac{\boldsymbol{x}_{k}}{\left|\boldsymbol{x}_{k}\right|^{3}}$.

where $\psi\left(x_{k}\right)$ is the sample selection function at distance $x_{k}$, whose inverse functions as weighting factor. For practical reasons, comparison with the inferred bulk flow $\boldsymbol{v}_{\text {bulk, }}$, we have translated the bulk acceleration into equivalent velocity units by means of the transformation $H f(\Omega, \Lambda) / \frac{3}{2} \Omega H^{2}$. The equivalent "discrete" expression for the external tidal shear is

$\mathcal{T}_{i j}=\frac{H f(\Omega, \Lambda)}{4 \pi n} \sum_{i} \frac{1}{\psi\left(x_{k}\right)} \frac{3 x_{k i} x_{k j}}{\left|x_{k}\right|^{5}}$.

For the $\Lambda$ CDM mock galaxy sample depicted in Fig. 16 we determine the gravity dipole by computing for a set of spherical external shells the resulting bulk flow acceleration (Eq. (36)) and the gravity quadrupole by computing external tidal shear (Eq. (37)). Recently, a similar approach was followed by Teodoro et al. (2004). The spherical shell volumes are defined by an inner radius $r_{\mathrm{inn}}=30 \mathrm{~h}^{-1} \mathrm{Mpc}$ and an outer radius $r_{\text {out }}$. The width of the shell is gradually enlarged by increasing $r_{\text {out }}$ from $r_{\text {out }}=30 h^{-1} \mathrm{Mpc}$ to $r_{\text {out }}=100 h^{-1} \mathrm{Mpc}$. The convergence of the resulting gravity dipole direction on the sky can be observed in Fig. 17. The small red diamonds are consistently located near $l \approx 230^{\circ}$, and converge at a sky location close to the direction of the velocity dipole (large red diamond). To get 


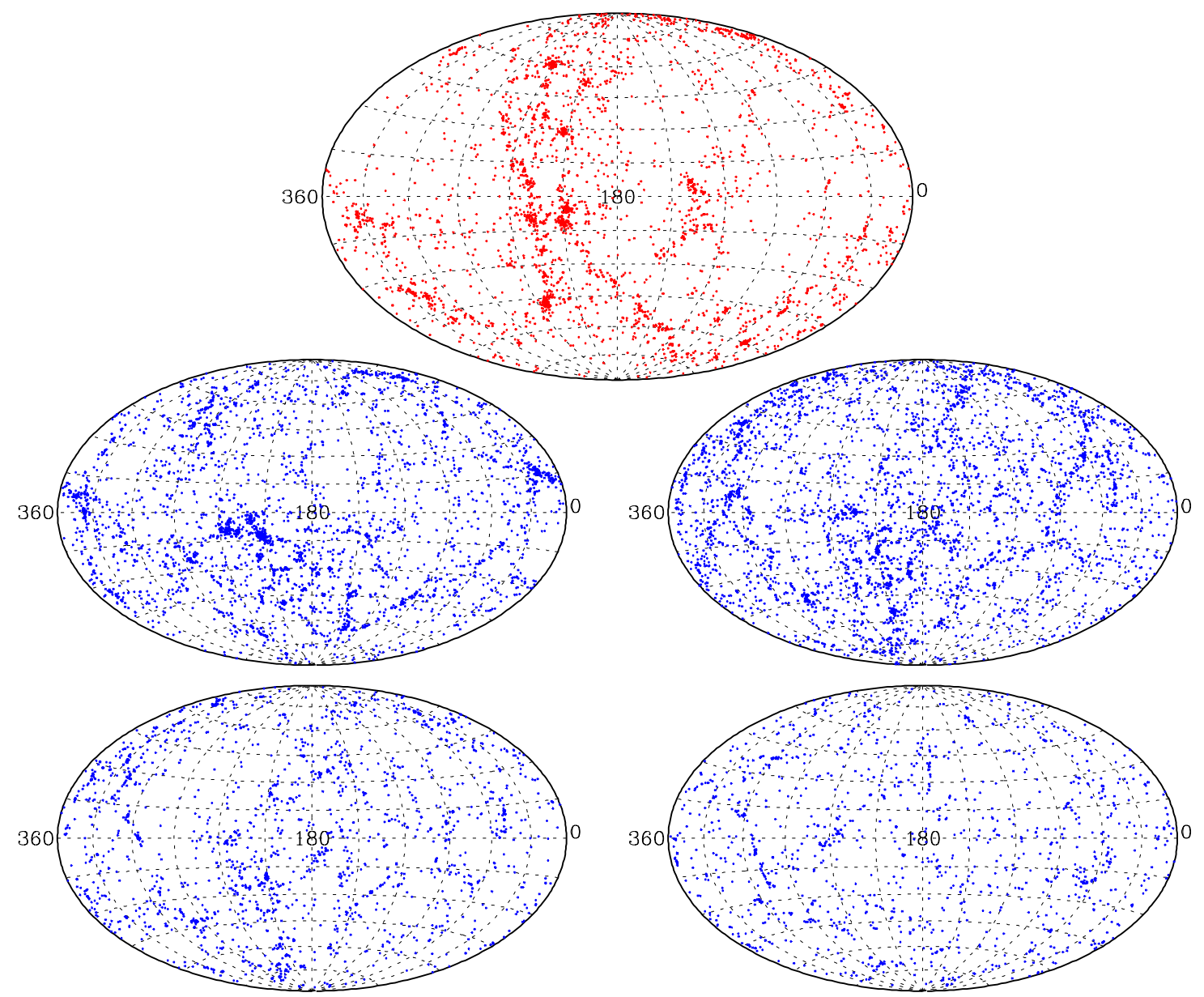

Fig. 16. Aitoff projections of the galaxy distribution of a NBG $+\mathrm{PSC} z \quad \Lambda \mathrm{CDM}$ catalog. The top-most panel shows the corresponding NBG distribution, $d_{\text {sur }}=[0,30] h^{-1}$ Mpc. The subsequent panels depict the external galaxy distribution enclosed by the shells defined by survey depth $d_{\text {sur }}=[30-55] h^{-1} \mathrm{Mpc}, d_{\text {sur }}=[55-70] h^{-1} \mathrm{Mpc}, d_{\text {sur }}=[70-85] h^{-1} \mathrm{Mpc}$ and $d_{\text {sur }}=[85-100] h^{-1} \mathrm{Mpc}$.

an idea of the amplitudes involved, Fig. 19 (top panel) shows the development of the cumulative gravity dipole as a function of external distance $d_{\text {sur }}\left(30 h^{-1} \mathrm{Mpc}<d_{\text {sur }}<100 h^{-1} \mathrm{Mpc}\right)$. By means of symbols the corresponding velocity dipole, for each of the three directions $x, y$ and $z$, are inserted at the outer radius of $d_{\text {sur }} \approx 100 h^{-1} \mathrm{Mpc}$. Note that we have restricted ourselves to a case study example. A more extensive and proper assessment, including a proper error estimate of both gravity dipole and quadrupole as well as the bulk and shear flow, is beyond the scope of the present argument. This issue, involving the shot noise influence on gravity dipole and quadrupole and the role of FAM uncertainties on the velocity flow components, will be treated in more detail in a forthcoming study.

We see that in the $x$-, $y$ and $z$-directions of the gravity and velocity dipoles are in reasonable agreement, within a margin of $\approx 30 \mathrm{~km} \mathrm{~s}^{-1}$. This observation justifies our expectation that the dipole can be estimated to sufficient accuracy from the surrounding external galaxy distribution. The dipole may then be estimated from the surrounding external galaxy distribution, so that the latter can be invoked to correct for the influence of the external tidal field in the dynamics of the local volume.

The situation is comparable for the cumulative tidal shear, in terms of its three eigenvalues and eigenvectors. Also the gravity quadrupole appears to converge relatively smoothly towards the velocity shear. This may be inferred from the plotted directions of the eigenvectors $\hat{\boldsymbol{e}}_{\mathcal{T} 1}, \hat{\boldsymbol{e}}_{\mathcal{T} 2}$ and $\hat{\boldsymbol{e}}_{\mathcal{T} 3}$ of the tidal shear $\mathcal{T}_{i j}$. They are indicated by means of three symbols, the triangle corresponding to the stretching component $\mathcal{T}_{1}$, the star the middle component $\mathcal{T}_{2}$ and the square the compressional component $\mathcal{T}_{3}$. The tidal shear tensor wanders extensively across the "sky" as we push the outer radius of the external shell outward, as is shown by the paths of the corresponding eigenvectors. Interestingly, once the shell radius starts to approach $100 h^{-1} \mathrm{Mpc}$, each of the eigenvectors appear to converge near the location of the corresponding stretching, central and compressing velocity shear tensor eigenvectors. However, also here we notice significant deviations in individual cases. 


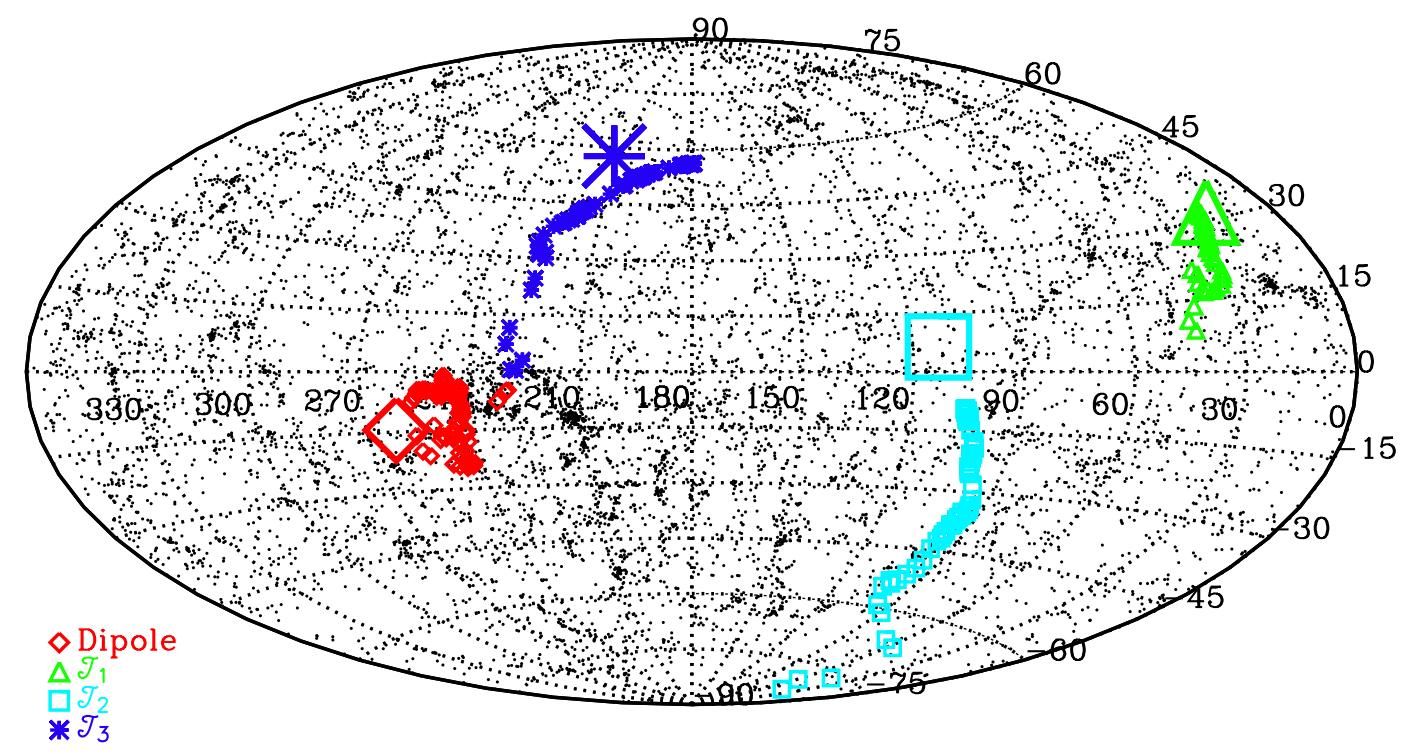

Fig. 17. Sky distribution of galaxies in external shell of survey depth $d_{\text {sur }}=30-100 \mathrm{~h}^{-1} \mathrm{Mpc}$. This galaxy distribution should reflect the mass distribution inducing the local tidal velocity flow. By means of symbols we have indicated the track of the gravity dipole and quadrupole eigenvector directions (cf. Eqs. (36) and (37)) on the sky, by radially expanding outward the survey depth $d_{\text {sur }}$ in steps of $1 h^{-1} \mathrm{Mpc}$, from $d_{\text {sur }}=30 h^{-1} \mathrm{Mpc}$ to $d_{\text {sur }}=100 \mathrm{~h}^{-1} \mathrm{Mpc}$. For comparison the same symbol, but then enlarged, indicates the directions for the corresponding bulk velocity and velocity shear eigenvector directions. Diamond: dipole. Triangle: stretching component shear. Square: middle component shear. Star: compressional component shear.

For comparison, we can appreciate the role of the external tidal field on local dynamics for the case of the $\tau \mathrm{CDM}$ cosmology. Figure 18 combines the galaxy sky distribution for a $\tau$ CDM mock galaxy sample, in the same radial shells as in Fig. 16. The final frame shows the Aitoff projection of the gravity dipole and gravity quadrupole eigenvectors for a set of gradually increasing radial shells. From the galaxy sky distribution in the four slices at sampling depths $d_{\text {sur }}=$ [0-30], [30-55], [55-70] and [85-100] $h^{-1} \mathrm{Mpc}$ we notice that these involve considerably more isotropic distributions. Hardly any prominent patterns can be discerned in the sky distribution. This is expressed in a more erratic wandering of gravity dipole and quadrupole directions (lower frame, Fig. 16). This also implies a more substantial contribution of shot noise effects. The latter represent a major source for deviations between the velocity dipole and shear flow quadrupole and the gravity dipole and quadrupole. The smaller coherence length of the $\tau \mathrm{CDM}$ fluctuations and the more randomly oriented contributions by the individual external matter concentrations may therefore be directly related to the lower level of coincidence between velocity and gravity directions than in the case of the more prominent anisotropies in the $\Lambda \mathrm{CDM}$ cosmology. In this, we have to realize that the amplitude of dipole and quadrupolar contributions between the two scenarios are not too different (cf. Table 5). The less prominent anisotropies in the $\tau \mathrm{CDM}$ catalogues are therefore compensated by a higher average matter density.

\subsection{Multipole components: summary}

The above results reassure the fact that the external tidal field can be well characterized by its main multipole components, the bulk flow and velocity shear. In terms of multipole amplitude convergence, these results show a better agreement for the $\Lambda \mathrm{CDM}$ model than for the $\tau \mathrm{CDM}$ one. This is due to the intrinsic characteristics of both cosmic models. As has been discussed in Sect. 4, and may be directly appreciated from Fig. 2, the relatively lower amplitude of the $\tau \mathrm{CDM}$ perturbations is compensated by a higher mass content. It leads to an equally strong external gravitational influence. On the other hand, the smaller spatial coherence of density features in the $\tau \mathrm{CDM}$ scenario causes the orientation of the gravity dipole and quadrupoles to be rather jittery. The direction of the cumulative gravitational force in the $\tau \mathrm{CDM}$ scenario wanders erratically over the sky as we move further out from the local volume. This differs from the situation in the $\Lambda$ CDM samples, where we observe a consistent, systematic and coherent convergence towards the final dipole direction.

The above results confirm the fact that the external tidal velocity field can be well characterized by its main multipole components, the bulk flow and velocity shear. This depends to some extent on the cosmology. In terms of multipole amplitude convergence, these results show a better agreement for the $\Lambda \mathrm{CDM}$ than for the $\tau \mathrm{CDM}$ model.

\section{Conclusions}

In this work we have applied the FAM technique to construct model velocity fields using mock catalogs resembling the NBG and IRAS-PSC $z$ galaxy catalogs. The mock catalogs were extracted from $N$-body simulations in which the central observer mimics some of the properties of the Local Group environment. Comparing FAM velocities obtained from the NBG mock catalogs with those obtained from the larger PSC $z$ mock catalogs and, finally, to the $N$-body velocities, allowed us to quantify the 

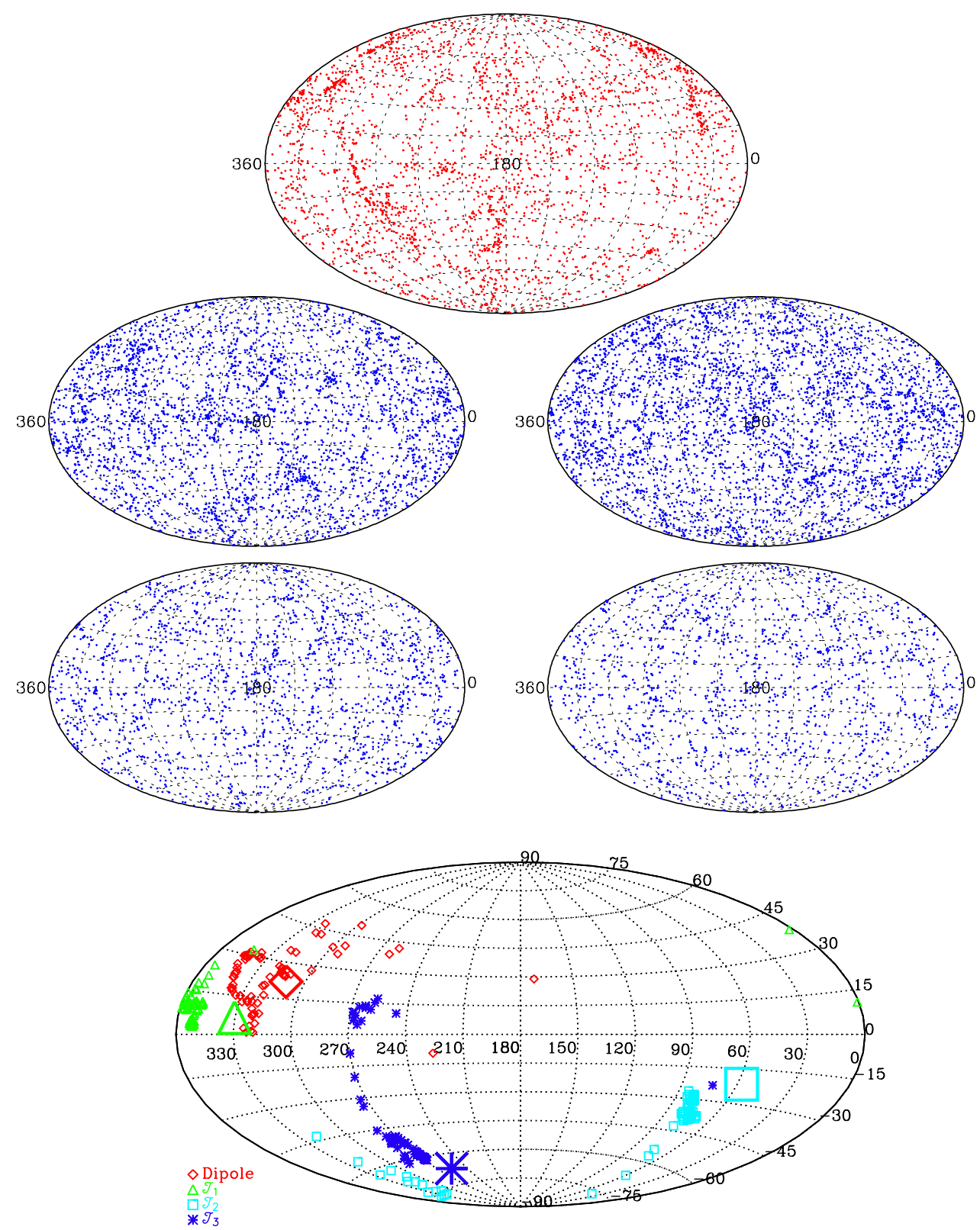

Fig. 18. Aitoff projections of the galaxy distribution of a $\mathrm{NBG}+\mathrm{PSC} z \tau \mathrm{CDM}$ catalog. The top-most panel shows the corresponding NBG distribution, $d_{\text {sur }}=[0,30] h^{-1} \mathrm{Mpc}$. The subsequent 4 panels depict the external galaxy distribution enclosed by the shells defined by survey depth $d_{\text {sur }}=[30-55] h^{-1} \mathrm{Mpc}, d_{\text {sur }}=[55-70] h^{-1} \mathrm{Mpc} d_{\text {sur }}=[70-85] h^{-1} \mathrm{Mpc}$ and $d_{\text {sur }}=[85-100] h^{-1}$ Mpc. Bottom panel: convergence gravity dipole and quadrupole. By means of symbols we have indicated the track of the gravity dipole and quadrupole eigenvector directions (cf. Eqs. (36) and (37)) on the sky, by radially expanding outward the survey depth $d_{\text {sur }}$ in steps of $1 h^{-1} \mathrm{Mpc}$, from $d_{\text {sur }}=30 h^{-1} \mathrm{Mpc}$ to $d_{\text {sur }}=100 h^{-1} \mathrm{Mpc}$. For comparison the same symbol, but then enlarged, indicates the directions for the corresponding bulk velocity and velocity shear eigenvector directions. Diamond: dipole. Triangle: stretching component shear. Square: middle component shear. Star: compressional component shear.

importance of the gravity field generated by the mass distribution within and beyond the LS.

Neglecting the mass distribution outside the LS leads to a systematic underestimate of the gravity field. The amplitude of this bias depends on the amount of power on scales larger than the LS, and thus on the cosmological models. In a $\tau \mathrm{CDM}$ universe model peculiar velocities are $\sim 20 \%$ smaller than the true ones. In the case of a $\Lambda \mathrm{CDM}$ model, which has more power on large scales, model velocities are underestimated by $\sim 35 \%$.

The results of the described FAM analyses are encouraging in the sense that the presently available all-sky, flux limited catalogs such as $\mathrm{PSC} z$ appear to be capable of 


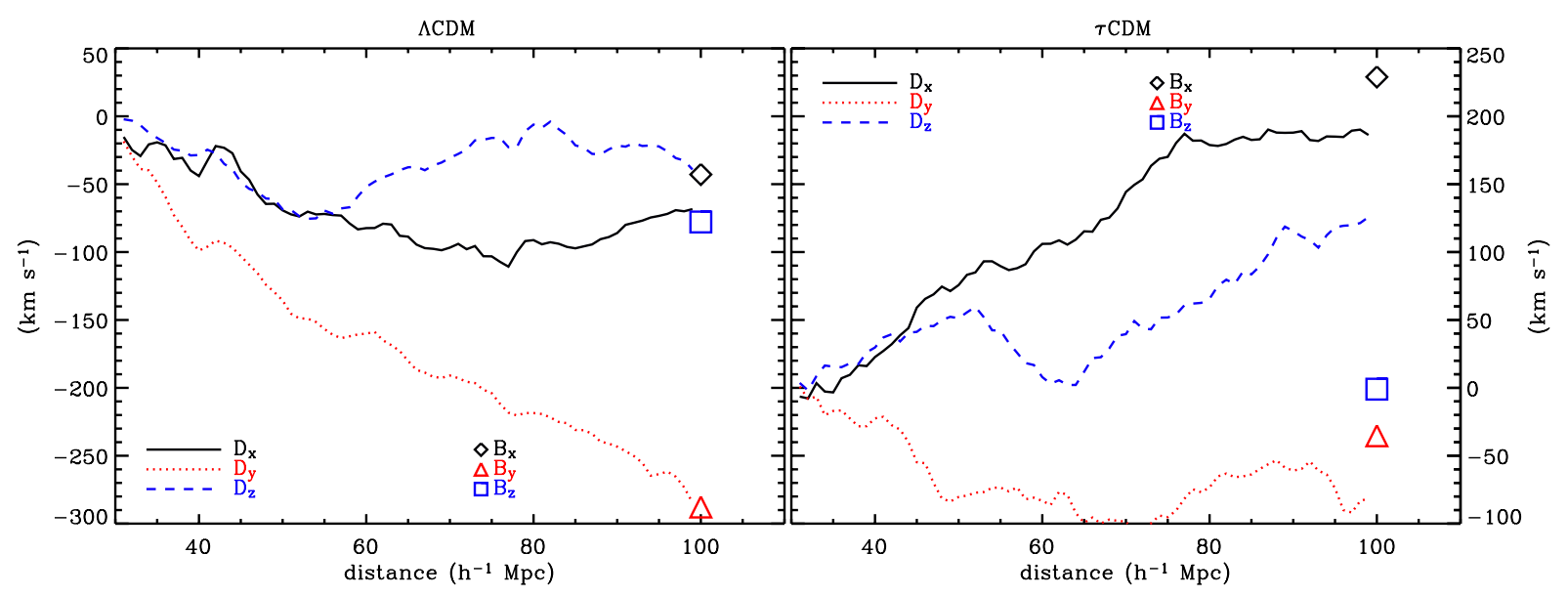

Fig. 19. Cumulative gravity dipole and tidal gravity shear. As a function of survey depth $d_{\text {sur }}$ the Cartesian components of the gravity dipole, $g_{x}, g_{y}$ and $g_{z}$ is followed (in equivalent velocity unit $\mathrm{km} \mathrm{s}^{-1}$ ). The symbols at $d_{\text {sur }}$ indicate the corresponding velocity dipole. Lefthand panel: $\Lambda$ CDM. Righthand panel: $\tau$ CDM.

accounting for the major share of the velocity field on the scale of the Local Supercluster. While the $30 h^{-1} \mathrm{Mpc}$ restricted NBG sample showed a substantial deficiency in its capacity to generate the local cosmic motions, in particular in the case of the $\Lambda$ CDM Universe models, in both cases the $100 h^{-1} \mathrm{Mpc}$ mock samples appear to embody nearly all matter concentrations responsible for the generated velocities in our local (NBG catalog) neighbourhood.

Also we notice a telling difference between the performance of both $\mathrm{FAM}_{30}$ and $\mathrm{FAM}_{100}$ reconstructions for the case of the $\Lambda \mathrm{CDM}$ cosmology catalogs on the one hand and the $\tau \mathrm{CDM}$ model catalogs on the other hand. The fact that the $\Lambda$ CDM model involves substantially more power on large scales, $r>30 h^{-1} \mathrm{Mpc}$, than the $\tau \mathrm{CDM}$ model is reflected in the better quality of the $\mathrm{FAM}_{30}$ reconstructions for the $\tau \mathrm{CDM}$ catalogs. The presence of substantial mass inhomogeneities with a scale in excess of that of the local Universe regions implies a larger external contribution to the local velocity field. This is also borne out by the fact that for the $\Lambda$ CDM catalogs we see a considerable improvement in velocity field reconstruction quality going from the $\mathrm{FAM}_{30}$ to the $\mathrm{FAM}_{100}$ reconstructions (see Table 4), while this is far less so for the $\tau \mathrm{CDM}$ catalogs.

Of course, whether the resulting models do indeed form an unbiased representation of the actual velocity field will to some extent also depend on whether the galaxy distribution in the flux limited galaxy catalogs does represent an unbiased reflection of the actual (external) mass distribution surrounding the Local Supercluster resembling region. The results of recent studies (Verde et al. 2002; Lahav et al. 2002; Tegmark et al. 2001; and Branchini et al. 2002) are quite encouraging in this respect. They seem to indicate, certainly on scales larger than $\approx 5 h^{-1} \mathrm{Mpc}$, that both IRAS and $2 \mathrm{dF}$ galaxies trace the underlying mass distribution in an unbiased fashion.

Nonetheless, observations along the lines of the presented mock catalogs seem to suggest that a proper analysis of Local Universe dynamics based on a combination of information of local small-scale (peculiar) galaxy velocities and a rough yet well-founded idea of the matter distribution on scales of a few hundred $100 h^{-1} \mathrm{Mpc}$ may help us towards acquiring far more insight into the dynamical history of the emergence and assembly of the striking nonlinear patterns we have discovered in the large scale matter distribution. Moreover, we have uncovered evidence that a meticulous point-to-point analysis of such velocity samples may help towards modelling the total local force field, including a proper model for the external forces.

When modeling the peculiar velocity of a LS look-alike region by only considering the matter distribution within $30 \mathrm{~h}^{-1} \mathrm{Mpc}$, the end product is a biased velocity field lacking of any large scale signature. This bias can be eliminated by accounting for the mass distribution beyond the LS. Our experiments demonstrate that sampling the mass distribution out to scales of $100 \mathrm{~h}^{-1} \mathrm{Mpc}$, in a flux limited fashion, is sufficient to account for the large scale contribution to the peculiar velocities in our cosmological neighborhood. More precisely, we have found that the cosmic velocity field within the LS, modeled by FAM using the mass distribution traced by PSC $z$ galaxies out to $100 h^{-1} \mathrm{Mpc}$ is unbiased. The differences between true and FAM velocity field are random and mainly occur in high density environments which are dominated by virial motions that are not modeled correctly by FAM.

The gravity and velocity fields generated by the mass distribution beyond the scale of the Local Supercluster are well characterized by their bulk flow and shear components. Therefore, one can obtain an unbiased model velocity field by superimposing a local model velocity field within the Local Supercluster to the bulk flow and shear components of the velocity field generated by the mass distribution between 30 and $100 h^{-1}$ Mpc.

These considerations suggest that velocity models which only consider the dynamics within the Local Superclusters might have been affected by systematic errors. In particular, our work suggests that, when compared with observed velocities, they might have underestimated the value of the density parameter, $\Omega_{\mathrm{m}}$, by $15-25 \%$. However, the analysis of Shaya et al. (1995), based on the galaxy distribution in the Local Supercluster, shows that a lower, not a larger, value of $\Omega_{\mathrm{m}}$ is found when complementing the local mass distribution with the large scale one traced by rich Abell clusters. 
A more precise evaluation of this bias will be performed in a future work in which we will perform the same analysis presented here using a new set of mock catalogs that are constrain to reproduce the distribution of the mass in our local Universe (see e.g. van de Weygaert \& Hoffman 1999; Mathis et al. 2002; Klypin et al. 2003).

Furthermore, our analysis shows that all model velocity fields of the Local Supercluster which are based on the PSC $z$ catalog (e.g. Branchini et al. 1999; Schmoldt et al. 1999; Valentine et al. 2000; Sharpe et al. 2001) are free from systematic biases arising from having neglected the large scale contribution from scales beyond its realm. Moreover, since the IRAS PSC $z$ survey is considerably deeper than $100 h^{-1} \mathrm{Mpc}$, it is reasonable to assume that the $\mathrm{PSC} z$ catalog can be used to predict unbiased velocities well beyond our Local Supercluster that, if compared with observed galaxy peculiar velocities, can discriminate among different cosmologies characterized by different values of $\Omega_{\mathrm{m}}$, like the $\Lambda \mathrm{CDM}$ and $\tau \mathrm{CDM}$ models.

The plausibility of this hypothesis has been recently confirmed by the analysis of Hoffman et al. (2001) that shows that the bulk and shear components of the external velocity field in the local universe inferred from the peculiar velocities in the Mark III catalog (Willick et al. 1997a,b) are qualitatively consistent with those expected from the mass distribution traced by IRAS PSC $z$ galaxies. On the other hand, the claim on the basis of the SMAC cluster peculiar velocity sample (Hudson et al. 2004) of an extra $225 \mathrm{~km} \mathrm{~s}^{-1}$ bulk flow component generated by matter concentrations on a scale exceeding $100 h^{-1} \mathrm{Mpc}$ should issue some caution with respect to claims of having accounted for all external influences on the local cosmic flow.

Coupling the local velocity model provided by FAM to the large scale contribution provided by linear theory allows to obtain a model velocity field which is unbiased, nonlinear and fast to compute. This means that, for the first time, we are in the position of performing a large number of experiments aimed at studying the nonlinear evolution of cosmic structures, such as filaments and clusters, and explore the role of tidal fields during their gravitational collapse. This relates to the observation that filaments are forming as a consequence of anisotropic collapse, induced a compressional tidal force acting perpendicular to the "axis" of the filament. By tracing out the coherent paths of the compressional modes of the primordial tidal field one can identify the sites of the later nonlinear filaments (Bond et al. 1996; see Van de Weygaert 2002). In turn this is directly related to cluster locations: the strong primordial tidal shear is the result of a local quadrupolar mass distribution. The corresponding overdensities tend to evolve into rich clusters, explaining the intimate link of clusters and filaments in the cosmic web.

Finally, it is worth stressing that in this work we have neglected the fact that we measure galaxy redshifts rather then positions. By means of an elegant formalism, Phelps (2000) demonstrated the feasibility of working out the action principle in redshift space. With respect to FAM, Nusser \& Branchini (2000) have shown that it can be easily implemented in redshift space and Branchini et al. (2002) demonstrated that it performs equally well in real and redshift space. Therefore, our unbiased, nonlinear model velocity field also allows to perform an accurate correction for redshift space distortions and thus lead to a precise reconstruction of the mass distribution in real space. Mapping the mass in the local universe down to nonlinear scales and comparing it with the distribution of baryonic mass (in form of stars or diffuse, ionized gas) is of considerable astrophysical interests as it will constrain and help understanding the process of galaxy formation and evolution within the Universe

Acknowledgements. The authors thank Shaun Cole for allowing the use of his $N$-body simulations. E.R.D. thanks W. E. Schaap for stimulating discussions. E.R.D. thanks the Universita' di Roma tre for its hospitality while part of this work was done. E.R.D. has been supported by The National Council for Research and Technology (CONACyT, México) through a scholarship. San Crispino provided unique and inspiring guidance.

\section{References}

Bardeen, J. M., Bond, J. R., Kaiser, N., \& Szalay, A. S. 1986, ApJ, 304,15

Berlind, A. A., Narayanan, V. K., \& Weinberg, D. H. 2000, ApJ, 537, 537

Bond, J. R., \& Efstathiou, G. 1991, Phys. Lett. B., 265, 245

Bond, J. R., Kofman, L., \& Pogosyan, D. Yu. 1996, Nature, 380, 603

Bouchet, F., \& Hernquist, L. 1988, ApJS, 68, 521

Branchini, E., \& Carlberg, R. 1994, ApJ, 434, 73

Branchini, E., Teodoro, L., Frenk, C. S., et al. 1999, MNRAS, 308, 1

Branchini, E., Eldar, A., \& Nusser, A. 2002, MNRAS, 335, 53

Branchini, E., Freudling, W., Da Costa, L. N., et al. 2001, MNRAS, 326, 1191

Branchini, E., Dekel, A., \& Sigad, Y. 2002, MNRAS, submitted

Carlberg, R., Couchman, H., \& Thomas, P. 1990, ApJ, 352, L29

Cole, S. 1997, MNRAS, 286, 38

Cole, S., Hatton, S., Weinberg, D. H., \& Frenk, C. S., 1998, MNRAS, 300,945

Colless, M., the 2dFGRS team 2003 [arXiv: astro-ph/0306581]

Colless, M. 2004, in Measuring and Modeling the Universe, ed. W. L. Freeman, Carnegie Obs. Astrophys. Ser., 2, in press [arXiv: astro-ph/0305051]

da Costa, L. N., Bernardi, M., Alonso, M. V., et al. 2000, AJ, 120, 95

Croft, R. A. C., \& Gaztañaga, E. 1997, MNRAS, 285, 793

Davis, M., Nusser, A., \& Willick, J. A. 1996, ApJ, 473, 22

Dekel, A. 1994, ARA\&A, 32, 371

De Lapparent, V., Geller, M. J., \& Huchra, J. P. 1986, ApJ, 302, L1

Dunn, A. M., \& Laflamme, R. 1993, MNRAS, 264, 865

Eke, V., Cole, S., \& Frenk, C. 1996, MNRAS, 282, 263

Faber, S. M., \& Burstein, D. 1988, in The Vatican Study Week on Large Scale Motions in the Universe, ed. G. V. Coyne, \& V. C. Rubin (Princeton: Princeton Univ. Press), 115

Fisher, K. B., Huchra, J. P., Strauss, M. A., et al. 1995, ApJS, 100, 69

Han, M., \& Mould, J. 1990, ApJ, 360, 448

Giavalisco, M., Mancinelli, P. J., Mancinelli, P. J., \& Yahil, A. 1993, ApJ, 411, 9

Giovanelli, R., Haynes, M. P., Herter, T., et al. 1997, AJ, 113, 22

Giovanelli, R., Haynes, M. P., Herter, T., et al. 1997, AJ, 113, 53

Goldberg, D. M., \& Spergel, D. N. 2000, ApJ, 544, 21

Hamilton, A. J. S., Tegmark, M., \& Padmanabhan, N. 2000, MNRAS, 317, L23

Hamilton, A. J. S., \& Tegmark, M. 2002, MNRAS, 330, 506

Haynes, M. P., Giovanelli, R., Salzer, J. J., et al. 1999, AJ, 117, 1668

Haynes, M. P., Giovanelli, R., Chamaraux, P., et al. 1999, AJ, 117, 2039 
Hoffman, Y., \& Ribak, E. 1991, ApJ, 380, L5

Hoffman, Y., Eldar, A., Zaroubi, S., \& Dekel, A. 2001 [arXiv: astro-ph/0102190]

Hudson, M. J., Lucey, J. R., Smith, R. J., Schlegel, D. J., \& Davies, R. L. 2001, MNRAS, 327, 265

Hudson, M. J., Smith, R. J., Lucey, J. R., \& Branchini, E. 2004, MNRAS, 352, 61

Jackson, J. D. 1975, Classical Electrodynamics, 2nd Ed. (Wiley)

Kaiser, N. 1991, ApJ, 366, 388

Klypin, A., Hoffman, Y., Kravtsov, A. V., \& Gottlöber, S. 2003, ApJ, 596, 19

Kolatt, T., Dekel, A., Ganon, G., \& Willick, J. A. 1996, ApJ, 458, 419

Lahav, O., the 2dFGRS team 2002, MNRAS, 333, 961

Lauer, T. R., \& Postman, M. 1995, ApJ, 425, 418

Lilje, P. B., Yahil, A., \& Jones, B. J. T. 1986, ApJ, 307, 91

Loveday, J., Maddox, S., Efstathiou, G., \& Peterson, B. A. 1995, ApJ, 442, 457

Lynden-Bell, D., \& Lahav, O. 1988, in The Vatican Study Week on Large Scale Motions in the Universe, ed. G. V. Coyne, \& V. C. Rubin (Princeton: Princeton Univ. Press), 199

Lynden-Bell, D., Faber, S. M., Burstein, D., et al. 1988, ApJ, 326, 19

Maddox, S. J., Efstathiou, G., \& Sutherland, W. J. 1996, MNRAS, 283,1227

Mathis, H., Lemson, G., Springel, V., et al. 2002, MNRAS, 333, 739

Maddox, S. J., Efstathiou, G., \& Sutherland, W. J. 1996, MNRAS, 283,1227

Nusser, A., \& Colberg, J. 1998, MNRAS, 292, 475

Nusser, A., \& Branchini, E. 2000, MNRAS, 313, 587

Nusser, A., da Costa, L., Branchini, E., et al. 2000, MNRAS, 320, L21

Peebles, P. J. E. 1980, The Large Scale Structure (Princeton, NJ: Universe. Princeton Univ. Press)

Peebles, P. J. E. 1989, ApJ, 344, L53

Peebles, P. J. E. 1990, ApJ, 362, 1

Peebles, P. J. E. 1994, ApJ, 429, 43

Peebles, P. J. E., Phelps, S. D., Shaya, E. J., \& Tully, R. B. 2001, ApJ, 554,104

Phelps, S. D. 2000, Ph.D. Thesis, Princeton Univ.

Phelps, S. D. 2002, ApJ, 575, 1

Press, W. H., Teukolsky, S. A., Vetterling, W. T., \& Flannery, B. P. 1992, Numerical Recipes (Cambridge: Cambridge University Press)

Santiago, B. X., Strauss, M. A., Lahav, O., et al. 1995, ApJ, 446, 457

Raychaudhury, S., \& Lynden-Bell, D. 1989, MNRAS, 240, 195

Sahni, V., \& Coles, P. 1995, Phys. Rep., 262, 1

Saunders, W., Sutherland, W. J., Maddox, S. J., et al. 2000, MNRAS, 317,55

Schmoldt, I. M., \& Saha, P. 1998, AJ, 115, 223
Schmoldt, I. M., Saar, V., Saha, P., et al. 1999, AJ, 118, 1146

Shandarin, S. F., \& Zel'dovich, Ya. B. 1989, Rev. Mod. Phys., 61, 185

Sharpe, J., Rowan-Robinson, M., Canavezes, A., et al. 2001, MNRAS, 322,121

Shaya, E. J., Peebles, P. J. E., \& Tully, R. B. 1995, ApJ, 454, 15

Shectman, S. A., Landy, S. D., Oemler, A., et al. 1996, ApJ, 470, 172

Strauss, M. A., \& Willick, J. A. 1995, Phys. Rep., 261, 271

Tegmark, M., Zaldarriaga, M., \& Hamilton, A. 2001, Phys. Rev. D, 63, 043007

Tegmark, M., Hamilton, A. J. S., \& Xu Y. 2002, MNRAS, 335, 887

Tegmark, M., Blanton, M. R., Strauss, M. A., et al. 2004, ApJ, 606, 702

Teodoro, L., Branchini, E., \& Frenk, C. S. 2004, MNRAS, in press [arXiv: astro-ph/0308027]

Tonry, J. L., \& Davis, M. 1981, ApJ, 246, 680

Tonry, J. L., Blakeslee, J. P., Ajhar, E. A., \& Dressler, A. 2000, ApJ, 530,625

Tormen, G., \& Bertschinger, E. 1996, ApJ, 472, 14

Tully, R. B., \& Shaya, E. J. 1984, ApJ, 281, 31

Tully, R. B. 1988, Nearby Galaxy Catalog, [NBG] (Cambridge: Cambridge University Press)

van de Weygaert, R., \& Bertschinger, E. 1996, MNRAS, 281, 84

van de Weygaert, R., \& Hoffman, Y. 1999, Evolution of Large Scale Structure, ed. A. Banday, R. Sheth, \& L. N. Da Costa (Garching: ESO), 178

van de Weygaert, R. 2002, Froth Across the Universe, Dynamics and the Stochastic Geometry of the Cosmic Foam, inv. review in Proceedings 2nd Hellenic Cosmology Workshop, ed. M. Plionis, S. Cotsakis, \& I. Georgantopoulos (Kluwer), ASSL, 276, 119

Valentine, H., Saunders, W., \& Taylor, A. 2000, MNRAS, 319, 13

Verde, L., the 2dFGRS team 2002, MNRAS, 335, 432

Volonteri, M., Haardt, F., \& Madau, P. 2003, ApJ, 582, 559

Webster, M., Lahav, O., \& Fisher, K. 1997, MNRAS, 287, 425

White, S. D. M., Efstathiou, G., \& Frenk, C. S. 1993, MNRAS, 262, 1023

Willick, J., \& Strauss, M. 1998, ApJ, 486, 629

Willick, J. A., Courteau, S., Faber, S. M., et al. 1997a, ApJS, 109, 333

Willick, J., Strauss, M., Dekel, A., \& Kolatt, T. 1997b, ApJ, 486, 629

Yahil, A., Strauss, M. A., Davis M., \& Huchra, J. P. 1991, ApJ, 372, 380

Zaroubi, S., \& Hoffman, Y. 1996, ApJ, 462, 25

Zaroubi, S., Hoffman, Y., Fisher, K. B., \& Lahav, O. 1995, ApJ, 449, 446

Zehavi, I., Blanton, M. R., Frieman, J. A., et al. 2002, ApJ, 571, 172

Zel'dovich, Y. B. 1970, A\&A, 5, 84 